\title{
Distribution System Services Provided by Electric Vehicles: Recent Status, Challenges, and Future Prospects
}

Arias, Nataly Bañol ; Hashemi Toghroljerdi, Seyedmostafa; Andersen, Peter Bach; Træholt, Chresten; Romero, Ruben

Published in:

IEEE Transactions on Intelligent Transportation Systems

Link to article, DOI:

10.1109/TITS.2018.2889439

Publication date:

2019

Document Version

Peer reviewed version

Link back to DTU Orbit

Citation (APA):

Arias, N. B., Hashemi Toghroljerdi, S., Andersen, P. B., Træholt, C., \& Romero, R. (2019). Distribution System Services Provided by Electric Vehicles: Recent Status, Challenges, and Future Prospects. IEEE Transactions on Intelligent Transportation Systems, 20(12), 4277-4296. https://doi.org/10.1109/TITS.2018.2889439

\section{General rights}

Copyright and moral rights for the publications made accessible in the public portal are retained by the authors and/or other copyright owners and it is a condition of accessing publications that users recognise and abide by the legal requirements associated with these rights.

- Users may download and print one copy of any publication from the public portal for the purpose of private study or research.

- You may not further distribute the material or use it for any profit-making activity or commercial gain

- You may freely distribute the URL identifying the publication in the public portal 


\title{
Distribution System Services Provided by Electric Vehicles: Recent Status, Challenges, and Future Prospects
}

\author{
Nataly Bañol Arias, Member, IEEE, Seyedmostafa Hashemi, Member, IEEE, Peter B. Andersen, \\ Member, IEEE, Chresten Træholt, Member, IEEE, and Rubén Romero, Senior Member, IEEE
}

\begin{abstract}
Trend-setting countries have promoted or even employed an increased number of electric vehicles (EVs) and other distributed energy resources (DERs) in their power systems. This development has triggered new and increasing challenges in the distribution system planning and operation, whereby distribution systems must adapt to the increased share of DERs. However, EVs may also offer new opportunities and can be used to support the grid by providing several local and global powerand energy-based services. This paper presents a review and classification of the services potentially available from EVs for distribution systems, referred to as EV distribution system services (EV-DSS). A detailed description of recent services and approaches is given, and an assessment of the maturity of EV-DSS is provided. Moreover, challenges and prospects for future research are identified, considering key topics, such as the design of the market framework, economic assessment, battery degradation, and the impacts of the transmission system operator service provision by EVs on distribution networks. Thus, this work offers a tool for stakeholders concerning services available from EVs and provides a broad literature framework that can be used as a base for further investigations. It is aligned with the current requirements to move toward realistic implementations of EV-DSS.
\end{abstract}

Keywords-Distribution Systems, Distribution System Services, Electric Vehicles, Renewable Energy Sources, Services Classification, Smart Grids.

\section{INTRODUCTION}

$\mathrm{R}$ ECENTLY, the concept of ancillary services for distribution networks has become a new paradigm in conjunction with the new roles assumed by distribution system operators (DSOs) in modern energy systems. With the advent of smart grids an increased number of distributed energy resources (DERs) in power systems has triggered new and increasing challenges in distribution system planning and

Manuscript submitted April 04, 2018. This work was supported by the Danish research Parker project under ForskEL contract no. 2016-1-12410 and by the Brazilian institution CAPES: PSDE, process no. 88881.135226/2016-01.

N. Bañol Arias and R. Romero are with the Electrical Engineering Department, UNESP - São Paulo State University, Ilha Solteira, CEP 15385-000, Brazil. (e-mail: natycanta@aluno.feis.unesp.br; ruben@dee.feis.unesp.br).

S. Hashemi, C. Træholt, and P. B. Andersen are with the Center for Electric and Energy, Department of Electrical Engineering, Technical University of Denmark (DTU), Kgs. Lyngby 2800, Denmark. (e-mail: shtog@elektro.dtu.dk; crt@elektro.dtu.dk; pba@elektro.dtu.dk).

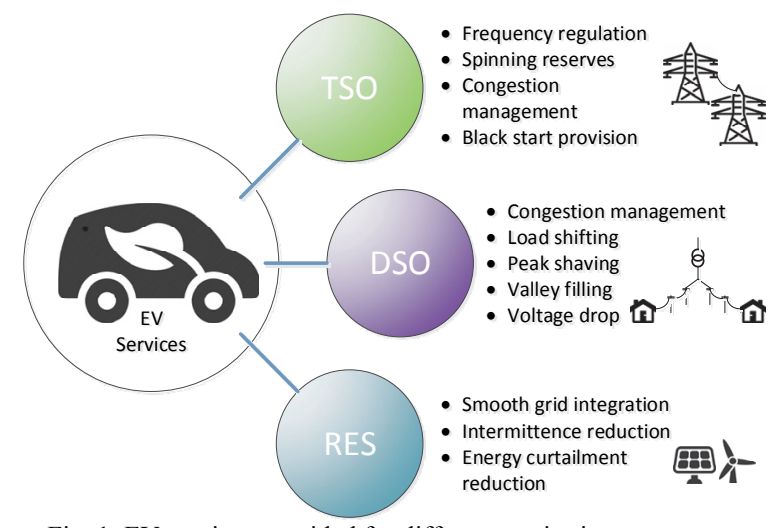

Fig. 1. EV services provided for different parties in power systems.

operation. Hence, distribution systems are required to assume new responsibilities and procure additional services to maintain high-quality services for end users.

These services can potentially be provided by batteries, including stationary batteries, and electric vehicles (EVs). An advantage of using stationary batteries is their permanent availability, which makes it possible to provide reliable services. However, the initial investment in stationary batteries is relatively high. EVs have the advantage in that they are purchased for a primary purpose, which is transportation, and that the service provision has second priority, but can offer alternative income for EV owners when the car is not being used for transportation.

Global EV penetration is increasing progressively. The increase is motivated by social, economic, and environmental factors, such as the support for transportation electrification from government policies, lower running costs, and the idea that EVs may address climate change and local air quality by reducing greenhouse gas emissions [1]. Consequently, it is anticipated that EVs will be integrated into the power systems in the future. Many studies have highlighted operational problems caused by EV integration into distribution systems. Increases in peak load, energy losses, overload of grid components, voltage drops, and, consequently, decreases in service quality are some of the adverse effects of a high EV penetration [2]-[4]. However, several works have been proposed in the literature to deal with these problems by using charging control algorithms and smart strategies in which EV charging is properly managed to decrease the challenges associated with EV integration [5]-[7]. 
EVs may also play an important role in the integration of renewable energy sources (RES) into distribution systems. Several studies have focused on the joint operation of EVs and RES facilitating smooth grid integration of intermittent renewable sources [8]-[10]. Although EVs are loads that have to be supplied by distribution systems, they may also offer new opportunities. Due to the manageable nature of their loads, they can be used to support the grid by providing several local and global power- and energy-based services. Fig. 1 illustrates some of the services that can be provided by EVs to different parties in the power system.

Most literature focuses on the services that EVs can provide for the transmission system operators (TSOs), usually called ancillary services. Frequency regulation, for instance, a service required by TSOs, has been widely addressed in the literature [11]-[16]. However, since the concept of services provided by EVs for DSOs is a new paradigm, most of the studies that have investigated distribution system services provided by EVs (EV-DSS) remain in theoretical applications.

EV-DSS are considered to be a set of flexible strategies that are provided to maintain an optimized and reliable operation of the local grid. In [17], the services that EVs can provide to DSOs, along with possible market structures, are discussed. In [18], a review on EV fleet management in smart grids is conducted. The authors develop a classification of different methods for the smart charging of EVs without focusing on EV-DSS. In [19], the feasibilities of certain services are evaluated for EVs in an aggregated mode, enabling DSOs to provide ancillary services for TSOs. In [20] and [21], the authors assess the framework, benefits, and challenges of vehicle-to-grid (V2G) technology and conduct a review of optimization techniques. [21] also includes a review of energy storage technologies deployed in EVs and the current regulations, standards, and issues within smart grids. Similarly, [22] and [23] review the EV technologies, their connectivity, impacts on the grid, benefits, and standards of EVs with RES in future smart grids. However, from the aforementioned works, some of the classifications are not clear about who receives more benefits from these services. Furthermore, there is no consistency in the name and definition of the EV services. By having a clear definition and classification of the services that EVs can provide at the distribution system level, it is possible to give EV-DSS a formal standing. Moreover, from the literature review, it is possible to identify that the requirements for a realistic implementation of EV-DSS have not been thoroughly investigated. We have identified that key topics, such as market framework design, economic assessment, battery degradation, the impact of TSO service provision on distribution networks, and emerging EV technologies and their impact in the distribution grid need further investigation to ensure an active and efficient participation of EVs in providing services for DSOs.

The primary goal of this paper is to identify the research gaps that need to be addressed to move toward a realistic implementation of EV-DSS. Aiming at completing the picture of potential EV-DSS, this paper contributes to the following. First, an up-to-date classification and description of the services that EVs can provide for distribution systems are presented as a first step to formally label EV-DSS. Second, a review of the recent literature is provided, including information regarding
V2G technology; methodologies and solution techniques; and consideration of the uncertainties, software, solvers, and characteristics of the test systems used in each approach. Third, a framework is presented comparing the work that has been done in the academic research with a real-life application using a specific case of frequency regulation. Finally, a comprehensive discussion is provided regarding the challenges and prospects of the EV-DSS, including key topics such as market framework, economic assessment, battery degradation aspects, impacts of the TSO service provision by EVs on distribution networks, and the impact of EV emerging technologies on the distribution grid. The information gathered in the second part provides an overview of the traditional methods and allows us to identify weakness in the control strategies as a means to encourage exploring new strategies aligned with the current requirements in realistic implementations of EV-DSS. The goal of the comparison in the third part is to assess the implementability of EV-DSS, i.e., how far the EV-DSS are from being implemented, and the stackability of EV-DSS, i.e., how easily EV-DSS can be combined with frequency regulation services. This is an innovative means to encourage the development of local DSO markets and promote the active participation of EVs as a service provider for DSOs.

Findings concerning battery degradation and the impact of the service provision to TSOs in distribution networks are supported by preliminary results obtained from the real implementation of the frequency-controlled normal operation reserve (FNR) provision to the TSO in Denmark [24]. This real implementation demonstrates that EVs are able to support power systems with their fast response; therefore, it is worth exploring the EVs' capability to support distribution systems. Accordingly, this paper contributes by encouraging new investigations on different aspects that remain unclear in order to move toward realistic implementations of EV-DSS.

The paper is organized as follows: Section II presents an overview of traditional classifications of EV services in power systems, and the proposed classification of the services provided by EVs specifically for DSOs is described. Section III describes the contributions of the reviewed works and discusses the weaknesses and research requirements. In Section IV, an assessment of the maturity of EV-DSS in real applications is carried out. Finally, challenges and future research suggestions are given in Section $\mathrm{V}$, followed by the conclusions provided in Section VI.

\section{OVERVIEW OF TRADITIONAL CLASSIFICATIONS OF EV SERVICES IN POWER SYSTEMS}

The concept of ancillary services appeared with the disaggregation of generation, transmission, and distribution systems, and it was mainly created for transmission systems, which are responsible for grid stability and security [25]. Ancillary services, along with the corresponding market framework, can vary among countries, due to the structural differences in their power systems [26], [27]. From the distribution system point of view, the concept of ancillary services provided to the DSOs is a new paradigm that has been emerging in conjunction with the new roles assumed by DSOs within the smart grid context. It appears as a set of flexible 
TABLE I

DIFFERENT CLASSIFICATIONS OF SERVICES PROVIDED BY EVS FOUND IN THE LITERATURE.

\begin{tabular}{|c|c|c|}
\hline Reference & Category & Service \\
\hline \multirow{2}{*}{ [18] } & Congestion prevention & Peak load reduction, power loss \\
\hline & Voltage regulation & - \\
\hline \multirow{6}{*}{ [19] } & Medium voltage grid & Peak power shaving, voltage regulation \\
\hline & Low voltage grid & Peak power shaving, voltage regulation \\
\hline & & Loss minimization \\
\hline & & Energy transmission cost minimization \\
\hline & High voltage grid & Frequency regulation \\
\hline & & Balancing mechanism (tertiary frequency control) \\
\hline \multirow{4}{*}{ [20] } & Power grid regulation & Frequency regulation \\
\hline & Spinning reserves & - \\
\hline & Active power support & Peak load shaving, load leveling, power loss \\
\hline & Reactive power compensation & Voltage regulation, power factor correction \\
\hline \multirow{3}{*}[23]{$^{*}$} & Load management & Load shifting \\
\hline & Power quality & Voltage regulation, load and voltage imbalance reduction \\
\hline & Power regulation & Frequency regulation, balancing power flow, absorb ramping power, stability \\
\hline \multirow{3}{*}{ [30] } & Frequency control & Regulation up and down, spinning and non-spinning reserve \\
\hline & Voltage control & - \\
\hline & System restoration & - \\
\hline \multirow{3}{*}{ [31] } & Frequency regulation/control & - \\
\hline & Loading services & Congestion prevention, loss reduction \\
\hline & Voltage service & Voltage magnitude regulation, imbalance reduction \\
\hline \multirow{7}{*}{ [33] } & & Frequency and voltage regulation \\
\hline & & Transient stability improvement \\
\hline & & Peak shaving \\
\hline & - & Valley filling \\
\hline & & Spinning reserve \\
\hline & & Power flow optimization \\
\hline & & Power quality improvement \\
\hline \multirow{4}{*}[34]{$^{*}$} & Spinning reserve & \\
\hline & Time shifting & \\
\hline & Active power support & - \\
\hline & Reactive power compensation & \\
\hline \multirow{4}{*}[35]{$^{* *}$} & Frequency regulation & - \\
\hline & Reserve & Spinning/non-spinning \\
\hline & Peak load leveling & - \\
\hline & Backup supply service & - \\
\hline \multirow{4}{*}[36]{$^{* *}$} & Frequency regulation & - \\
\hline & Spinning reserve & - \\
\hline & Supplemental Reserves & Non-spinning reserves \\
\hline & Replacement Reserves & Contingency reserve \\
\hline
\end{tabular}

services that can be provided to deal with the operational challenges (i.e., the challenges arising from the integration of EVs and RES into the grid). Because the concept of ancillary services is mostly related to the services provided for the transmission system, this paper will refer to services provided for the distribution systems as distribution system services (DSS). The use of DSS may help prevent or defer grid upgrade, but may also improve the operation of the grid through better management [28], [29].

Table I shows a summary of different classifications for the services provided by EVs in power systems, based on the literature. This table is constructed from papers in which a specific classification of EV services has been described. Each paper proposed a classification including different categories and services, which are named according to each author's preferences. Table I presents a traditional idea of grid services and shows how people usually classify grid services. In [20], the authors establish the frequency regulation service as part of the power grid regulation category. Peak load shaving, load leveling, and power loss services are considered in the category of active power support, whereas voltage regulation and power factor correction are services considered in the category of reactive power support. In [30], the authors consider up-down regulation and spinning and non-spinning reserve services in the frequency control category. The classification proposed in
[31] includes congestion prevention and loss reduction in the category of loading services, while voltage magnitude regulation and imbalance reduction are considered in the voltage services category. Similarly, in [18], peak load reduction and power loss services are considered in the congestion prevention category. The authors in [19] classify peak power shaving and voltage regulation for both medium and low voltage networks. The authors also propose an ancillary service called balancing mechanism, which is also found in the literature as a tertiary reserve [32]. Meanwhile, the authors in [33] do not specify categories; instead, they mention services such as frequency and voltage regulation, transient stability improvement, peak shaving, valley filling, spinning reserve, power flow optimization, and power quality improvement.

Some classifications found in the literature do not specify if the services are proposed for providing support to the DSOs or to the TSOs, as presented in [23] and [34]. Some references clearly specify if the services are proposed for supporting the transmission system, as presented in [35] and [36]. Moreover, in most of the studies related to ancillary services, it is difficult to identify who receives the most benefits: DSOs or TSOs.

Based on the classifications in Table I, one can notice that there is neither an agreement nor a clear classification of the EV services provided for DSOs. There is no consistency in the 
TABLE II

PROPOSED CLASSIFICATION OF THE EV-DSS.

\begin{tabular}{|c|c|c|c|}
\hline Category & Services & Related works & Alternative names \\
\hline \multirow{5}{*}{$\begin{array}{l}\text { Active power } \\
\text { support }\end{array}$} & Congestion management & [58], [59], [60], [61], [62], [63], [64], [65], [66], [67], [68], & Congestion alleviation, congestion prevention, \\
\hline & Loss minimization & $\begin{array}{l}\text { [19], [64], [69], [70], [71], [72], [73], [74], [81], [84], [96], } \\
{[107],[111]}\end{array}$ & Loss reduction \\
\hline & Load shifting & {$[72],[75],[76],[77],[78],[79],[80],[111]$} & Load leveling, load shaping, load flattening \\
\hline & Peak shaving - valley filling & [19], [67], [81], [82], [83], [84], [85], [86], [87], [88], [89], [90] & $\begin{array}{l}\text { Load shedding, load curtailment, peak clipping, } \\
\text { load variance, load shifting }\end{array}$ \\
\hline & Voltage control by active power & $\begin{array}{l}\text { [9], [19], [58], [59], [63], [64], [65], [70], [71], [80], [81], [91], } \\
{[92],[93],[94],[95],[96],[97],[110]}\end{array}$ & Voltage regulation, voltage deviation reduction \\
\hline \multirow{2}{*}{$\begin{array}{l}\text { Reactive power } \\
\text { support }\end{array}$} & Reactive power compensation & $\begin{array}{l}\text { [84], [98], [99], [100], [101], [102], [103], [104], [105], [106], } \\
{[107],[108],[109],[110]}\end{array}$ & \\
\hline & Voltage control by reactive power & $\begin{array}{l}{[10],[69],[84],[98],[101],[102],[103],[104],[105],[106],} \\
{[107],[109],[110],[111]}\end{array}$ & Voltage regulation \\
\hline \multirow{2}{*}{$\begin{array}{l}\text { Renewable energy } \\
\text { sources } \\
\text { integration } \\
\text { support }\end{array}$} & $\mathrm{EV}+\mathrm{PV}$ & [9], [10], [78], [96], [97], [107], [121], [122], [123], [124] & \\
\hline & $\mathrm{EV}+\mathrm{Wind}$ & [10], [109], [121] & \\
\hline
\end{tabular}

name and definition of the EV services. Frequency regulation, for instance, is a service mainly offered for the transmission system [35], [37]-[41]. Nonetheless, some works mention that this service could be required in the future for DSOs [18], [30]. In that case, the EV control for frequency regulation in microgrids could be also considered as a service for DSOs, since they have the potential to improve microgrid stability when operating in an isolated mode, as discussed in [42]-[45]. Most of the papers do not specify which kind of regulation is studied. Instead, the regulation services are mentioned in different ways, such as load regulation or load following. Non-spinning reserves are also found as supplemental reserves, while replacement reserves and contingency reserves refer to the same service.

Based on the previous findings of the works presented in Table I, it is possible to observe that a) Most of the studies that have investigated services provided by EVs are addressed to services mainly provided to TSOs and not at the distribution system level; b) The current classifications of EV services are not clear about which stakeholder receives the benefits of these services; c) There is no clarity among the names given to different services, which makes it difficult to provide a consistent EV-DSS portfolio. The definitions of the offered services vary from market to market, and the classification of services depends mainly on the particular requirements of each DSO and the regulations of each country. For this reason, we proposed our own classification of EV-DSS, as a first step to move toward the formalization of DSO products. Having a clear definition and classification of the services that EVs can provide at the distribution system level makes it possible to identify the current requirements for realistic implementations. In the following sections, the proposed classification of the services provided by EVs specifically for DSOs will be presented.

\section{A. Proposed classification of distribution system services provided by EVS}

Table II presents the proposed classification of EV-DSS in which the services are classified into three main categories: active power support, reactive power support, and RES integration support. This table includes a list of references from the specialized literature, in which each service has been addressed. It also includes a list of some alternative names found in the literature for each service.

Active power or real power is the main source of revenue for DSOs, and it has become an important issue for distribution systems. Usually, active power accomplishes the useful work, while reactive power supports voltage. However, if both active and reactive power are not efficiently controlled, operational/technical problems (e.g., overloading, overvoltage, undervoltage, and power loss increases) may occur. Therefore, active and reactive power should be efficiently controlled in order to maximize the earnings of DSOs while maintaining system reliability.

Congestion management, loss minimization, load shifting, peak shaving, valley filling, and voltage control are involved in the category of active power support services (frequency regulation service is not included in this category because it is typically a service required by TSOs). Most of the services included in the active power support category are part of a large concept known as demand side management. Demand side management is a methodology in which the demanded energy is modified through different methods and incentives. Thus, demand is redistributed and spread more uniformly during the planning horizon. Demand side management comprises several initiatives and methods [46]-[48], which are depicted in Fig. 2.

Note from Fig. 2 that demand response appears as an initiative of demand side management, which aims to induce lower electricity consumption by changing the normal consumption patterns of demand-side resources in response to changes in the price of electricity, or incentive payments [49]. Demand response can be accomplished by price signals (indirect load control) or predetermined contracts (direct load control) [50], applying methods such as load shifting. Peak shaving and valley filling are also among the methods used in demand side management. However, they can also be found in the literature as complements to the load shifting method, pursuing similar objectives, such as reducing peak demand, reducing energy cost, and avoiding violations of technical limits. For this reason, peak shaving and valley filling are connected to load shifting through the blue arrow in Fig. 2. Representations of peak shaving, valley filling, and load shifting are illustrated in Fig. 3 [51].

Reactive power compensation and voltage control or voltage 


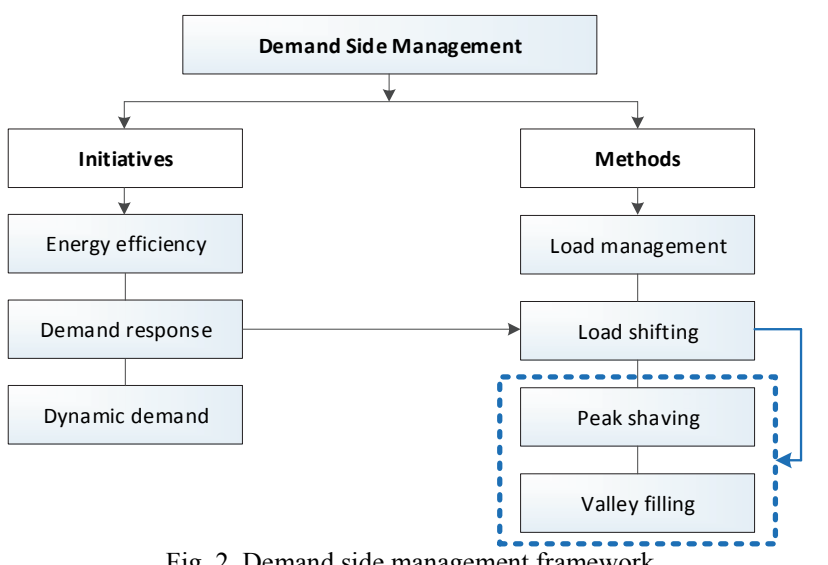

Fig. 2. Demand side management framework
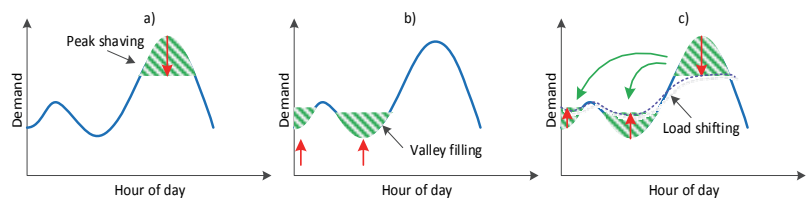

Fig. 3. Active power services a) Peak shaving b) Valley filling c) Load shifting

regulation are involved in the category of reactive power support services. Note that voltage regulation support is a service included in both categories: active and reactive power support.

Regarding the services provided by EVs for supporting RES integration into the grid, the main service found in the literature is the coordination between the operation of EVs and RES, which is divided into two subcategories: the charging and/or discharging of EVs, along with photovoltaic energy (EV+PV) and charging, and/or the discharging of EVs along with wind energy (EV+Wind).

It is noticeable that a relationship between the services regarding active power support exists, as they pursue similar objectives. For instance, congestion management can be addressed by using load shifting in an indirect way. Peak shaving and load shifting are similar services. Load shifting is performed by moving the load from peak to valley times, achieving shaving and filling. However, in load shifting, the load is kept as part of the global demand, whereas in peak shaving, it is removed [52]. Some of the papers found in the literature address active power support services using different names. For instance, some studies address load shifting, load leveling, or load shaping, the last being the most generic term. Nonetheless, they may have different purposes. In energy storage applications, for instance, load shifting has the purpose of reducing peak demand, while load leveling has the same purpose, but for an economic operation [53]. Additionally, it was found that the term load leveling is used more frequently on the supply side, i.e., by electric utility companies, while peak shaving is used more frequently on the demand side, i.e., by electric utility customers [54]. The valley filling service can also be found as load variance or load shifting. Most of these services deal with peak events and prevent network overloading by reducing the peak power demand. Furthermore, they not only lower utility bills, but also reduce technical problems on the power grid. Therefore, each service is taken into account separately in order to present a clear classification of the EV-DSS

The main contributions of each paper, along with the basic definitions and formulations of the services, are described in the next section. This material provides a guide for the reader regarding the approach to choose, depending on the EV-DSS under consideration.

\section{CONTRIBUTION OF THE REVIEWED WORKS}

In this section, the main contributions of the reviewed papers about EV-DSS are presented. Most of the reviewed papers are taken from recent literature. Table III shows a summary of the characteristics of the reviewed papers, including information related to the consideration of V2G technology, solution techniques applied to address the services, type of control (centralized or decentralized), and consideration of uncertainties within the methodology. Table III also includes information regarding the test system (e.g., number of buses, real or adapted systems, and one- or three-phase systems) and the type of software and solver used in the simulations. The list of papers is sorted chronologically. After classifying and describing the studies that have been done in the academic research in regard to EV-DSS, the weaknesses of the developed strategies and research requirements are identified and discussed.

\section{A. Active power services}

The EV demand could be considered a controllable load that can be managed for different purposes. It has been demonstrated that EVs are usually charged during the peak hours [55], when the price of electricity is high. Some services, such as load shifting, peak shaving, and valley filling, have been developed in order to solve congestion problems in the grid.

\section{1) Congestion management}

The concept of congestion in distribution systems typically appears when generation or demand exceeds the capacities of the installed equipment in the network, resulting in technical problems, such as overloading and power losses. Congestion management appears as a solution to deal with these issues [56]. It can be addressed in different manners, by using constrains for the thermal capacity of the installed equipment or establishing the capacity as an objective function. For instance, equation (1) represents a constraint to avoid overloads in lines; $P_{l}$ and $P_{l \max }$ are the power flow and thermal capacity of line $l$, respectively. In contrast to (1), (2) represents the total overload as a single objective function in which $N L, L F_{l}$, and $L_{c a p l}$ are the number of lines, the power flow, and the thermal capacity of line $l$, respectively [57].

$$
\begin{gathered}
\left|P_{l}\right| \leq P_{\text {lmax }} \\
\min \sum_{l=1}^{N L}\left(L F_{l}-L_{c a p l}\right)
\end{gathered}
$$

Congestion management is addressed in [58]-[68]. In [58], the authors develop a smart charging controller that can be used for centralized/decentralized EV control, helping the grid with congestion and frequency issues. The authors in [64] develop 
TABLE III

CHARACTERISTICS OF THE REVIEWED PAPERS ON EV-DSS

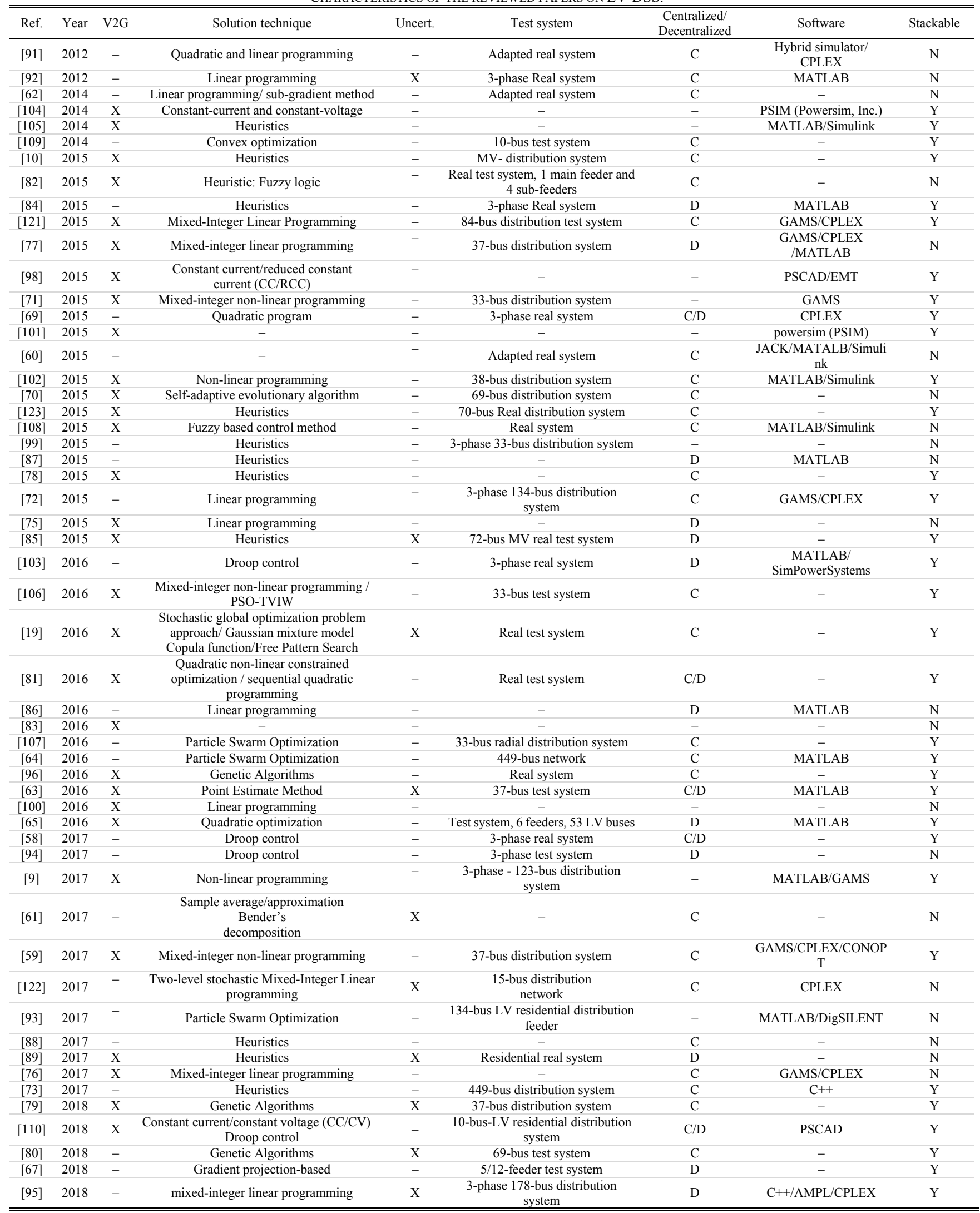

Information not available (-), Centralized methods (C), Decentralized methods (D), Low Voltage Network (LV), Yes (Y), No (N) 
TABLE III

CHARACTERISTICS OF THE REVIEWED PAPERS ON EV-DSS. (Continuation...)

\begin{tabular}{|c|c|c|c|c|c|c|c|c|}
\hline Ref. & Year & V2G & Solution technique & Uncert. & Test system & $\begin{array}{l}\text { Centralized/ } \\
\text { Decentralized }\end{array}$ & Software & Stackable \\
\hline [111] & 2018 & - & Non-linear programming & - & 33-bus distribution system & $\mathrm{C}$ & GAMS/KNITRO & $\mathrm{Y}$ \\
\hline [124] & 2018 & - & mixed-integer linear programming & - & $\begin{array}{c}\text { 107-bus distribution system (11 MV } \\
\text { and } 96 \mathrm{LV})\end{array}$ & $\mathrm{C}$ & AMPL/CPLEX & Y \\
\hline [74] & 2018 & $\mathrm{X}$ & Heuristics & - & Real distribution system & $\mathrm{C}$ & $\begin{array}{l}\text { MATLAB/DigSI } \\
\text { LENT }\end{array}$ & $\mathrm{Y}$ \\
\hline [68] & 2018 & - & linear programming & - & 4-bus test system & $\mathrm{D}$ & GAMS/CPLEX & Y \\
\hline [66] & 2018 & - & mixed-integer linear programming & $\mathrm{X}$ & 33-bus distribution system & $\mathrm{D}$ & GAMS/CPLEX & $\mathrm{Y}$ \\
\hline
\end{tabular}

Information not available (-), Centralized methods (C), Decentralized methods (D), Low Voltage Network (LV), Yes (Y), No (N)

an online algorithm for optimal EV charging coordination considering EV user preferences and grid technical conditions, contributing indirectly with loss minimization reduction.

In [67], a gradient projection-based method to deal with overloading on the transformers is proposed. Similarly, in [63], a probabilistic model is proposed to reduce the probability of congestion of lines and under/overvoltage in the nodes in a radial distribution network. The authors in [59] propose three different EV charging coordination algorithms to prevent line congestion, voltage drops, and transformer congestion.

A similar approach can be found in [62], in which the congestion problem is addressed through a coordination strategy to minimize the charging cost of EVs while maintaining thermal limits and considering the EV user requirements.

In contrast to the studies described above, in [61], an EV charging coordination model is proposed to control the EV loads at a public station with fast chargers installed. The EV load is managed using its charging flexibility, so congestion problems are avoided. Moreover, in [66] and [68], price-based methods are proposed for congestion management in distribution networks under high penetration of EVs and heat pumps.

\section{2) Loss minimization}

Power losses are the energy dissipated as heat through the conductors. Typically, as demand increases, power losses increase as well. A classic formulation for minimizing the power losses in distribution systems is given by (3), where $R_{l}$ and $I_{l}$ are the resistance and the current of line $l$, respectively.

$$
\min \sum_{l}^{N L} R_{l} I_{l}^{2}
$$

The strategies developed in [69]-[72], [73], and [74] deal with power losses in the grid. A multi-objective methodology for the day-ahead active and reactive power scheduling problem is proposed in [71]. Here, EVs contribute with voltage regulation through active power control. In [72], the proposed online EV charging coordination minimizes the total cost of the electricity and the power losses, considering the network security, power quality, and EV constraints. Load shifting is implicitly addressed in this work.

Centralized and decentralized EV charging coordination strategies for balancing the operation of distribution networks using EV chargers and PV inverters are proposed in [69]. The objective function aims to minimize the load variance, dealing with power losses and avoiding the violation of voltage limits. Distinct from [69], in [70], the authors propose a stochastic optimization model to solve the distribution feeder reconfiguration problem as a reliability-enhancing strategy to coordinate the V2G provision of EV fleets. In [73], charging coordination strategies are proposed to minimize the total operational cost of the distribution system considering customer preferences and to maintain the technical grid constraints, while in [74], the strategies are developed to minimize variations in the average voltage of the feeders.

\section{3) Load shifting}

Load shifting reduces the utility loads during periods of peak demand and builds the load in off-peak periods, which means that some appliances (e.g., EVs) are switched off during peak demand [51]. As a result, the electricity consumption is shifted from peak to off-peak hours, and vice versa.

Several studies have involved load-shifting strategies for minimizing the peak demand. For instance, in [75], the authors propose distributed algorithms that enable EV users to participate in demand response programs to reduce the peak demand and to flatten the demand curve through a game theory approach. Similarly, an intelligent algorithm to control EV charging/discharging is proposed in [76]. There, the algorithm takes advantage of the energy store capacity of EVs to provide support to the grid and avoids new peak demands by using a tariffs scheme.

The work presented in [77] proposes a mathematical model in which the EV load is shifted according to the charging prices, and the profit of all the involved parties is maximized. In the same way, in [78], an EV charging/discharging control for load leveling to minimize the electricity cost is proposed. The methodology is developed for peak-load shifting by using the EV batteries and PV panels to support electricity in a small-scale energy management system. In [79] and [80], smart charging strategies are proposed to deal with peak loads through load shifting. Although [79] considers the technical constraints of the grid and the EVs, [80] is more focused on economical solutions for future smart cities.

4) Peak shaving and valley filling

Peak shaving consists of the peak demand reduction of the system by direct load control, while valley filling is another form of load management, which embraces building load in off-peak periods and improving the load factor [51]. In terms of EVs, users are encouraged to charge their cars during the off-peak periods. Basic formulations for these services are presented in (4) and (5).

$$
\min \sum_{n=1}^{N} C_{n} \Delta P_{l c n}
$$




$$
\max \sum_{t \in T_{o f f-p e a k}} P_{t}
$$

In (4), the total curtailed load cost is minimized, where $N$ is the number of loads to be disconnected, $C_{n}$ is the cost of each load, and $\Delta P_{l c n}$ is the quantity of load curtailed. In (5), the power consumed from the grid at time $t\left(P_{t}\right)$ during the off-peak periods $\left(T_{\text {off-peak }}\right)$ is maximized. Several works have been developed in order to provide peak shaving and valley filling services [81]-[83]. Alternatively, [19], [84], and [85] only apply peak shaving, while in [86], [87], [88], [89], and [90], only valley filling is considered. In [82], the authors design fuzzy logic controllers to be used in the substations and in the EV charging stations for real-time EV charging coordination to support the grid. In [81] and [85], distributed EV charging strategies are proposed to minimize the system load variance and to flatten the daily grid demand. While [81] focuses on the EV/RES coordination, [85] focuses on the EV owners' preferences. A similar approach can be found in [83], in which an algorithm for EV charging/discharging coordination is proposed to minimize the peak load demand and to foresee congestion in the feeders and transformers. In [90], a strategy to coordinate the available PEV battery capacity for local peak shaving, along with a peak-shaving index, are proposed.

The work presented in [19] proposes an approach for the statistical assessment of the potential of plug-in EV fleets providing ancillary services using V2G technology. In [86], decentralized algorithms with minimal communication and delay considerations are proposed to prevent overloading in transformers and to achieve a desirable level of valley filling in the grid. Likewise, in [87], the authors propose strategies for solving the EV charging coordination problem, considering the EV owners' preferences while minimizing the variance and peak of the aggregated load profile. In [88], the authors propose a valley filling strategy for EV charging coordination that can improve the grid operation with a low computational algorithm, while in [89], EV charging/discharging control strategies are proposed using a predictive control approach to reduce power fluctuations from EV charging and their impact on the demand curve. In [89], the EV load is managed in the valley filling operation, similar to the approach proposed in [67]. Additionally, the works presented in [19], [81], and [84] contribute indirectly with loss minimization and voltage regulation.

\section{5) Voltage regulation by active power control}

Voltage issues appear when connecting generation or consumption units to the distribution network, leading to voltage rise or drop problems. Active and reactive voltage control methods have been developed to deal with these problems by controlling different network resources, such as on-load tap changers, distributed generation units, capacitor banks, and other controllable devices (e.g., EVs). Voltage control can be carried out using constraints for voltage limits as described in (6), where $V_{i}, V_{i}^{\min }$, and $V_{i}^{\max }$ are the voltage magnitude and the minimum and the maximum voltages at node $i$, respectively. It can also be formulated as an objective function in which the total voltage deviation, i.e., difference between a voltage reference and an estimated value, is minimized, as described in (7). $N_{b u s}, V_{i, r}$, and $V_{i}$ are the number of nodes, the voltage reference, and the estimated voltage at note $i$, respectively.

$$
\begin{aligned}
& V_{i}^{\text {min }} \leq V_{i} \leq V_{i}^{\text {max }} \\
& \min \sum_{l=1}^{N \text { bus }}\left(V_{i, r}-V_{i}\right)^{2}
\end{aligned}
$$

Voltage regulation has been addressed in [9], [65], [91]-[94], and [95]. In [9], a methodology for EV and PV coordination in real-time is developed in order to mitigate the imbalance in low voltage (LV) distribution grids by using V2G. In [9], demand response is used as a strategy to cope with peak demand and voltage drop issues. The study in [91] aims at minimizing the total EV charging cost, considering the interaction between the aggregator, the DSO, and the electricity market, while maintaining the technical conditions of the grid. In the same way, control algorithms are proposed in [92] to optimize the EV charging coordination, maximize the power required for each $\mathrm{EV}$, and maintain the grid operational constraints.

A method for optimal EV charging/discharging coordination to improve the voltage imbalance factor is proposed in [93], while the authors in [94] develop a methodology to improve the power quality of a low voltage network. A local smart charging controller is used for validating the EVs' ability to reduce voltage imbalances by modulating their charging current according to local voltage measurements. Similarly, in [95], a novel method is proposed that only requires local voltage magnitude measurements to maintain the operational limits of the distribution system. A similar approach can be found in [65], in which an algorithm for managing EV charging/discharging in LV distribution networks is proposed. The objective function aims at controlling the voltage profile while considering the EV users' preferences. Furthermore, the algorithm reduces the line and transformer loadings during the peak hours in an indirect way. In [19], [58], [59], [63], [64], [71], [70], [80], [81], [96], and [97], the voltage regulation service is indirectly addressed as an active power support service.

Most of the studies that address services in the category of active power support consider V2G technology in their approaches. Nonetheless, it is not a requirement to offer this kind of service to the grid.

\section{B. Reactive power services}

Reactive power and voltage control can be properly managed and implemented to provide adequate service quality. Several works have demonstrated that reactive power control can be used to maintain the voltage within acceptable levels. It can also be used to reduce power losses and congestion issues and to improve the power factor and congestion.

\section{1) Reactive power compensation}

Reactive power compensation is a method for balancing the capacitive and inductive components of a power system in order to provide sufficient voltage support and to enhance the reliability and security of the grid. In terms of EVs, it is a service provided by the DC-link capacitor of an EV bidirectional battery charger, which, according to the literature, could be advantageous in terms of the life cycle of the battery because the battery is not directly responsible for providing this 
service [20]. Different studies addressing this topic can be found in [84], [98]-[108], and [109]. Furthermore, most of them also deal with issues related to voltage drops and power losses. For instance, in [84], despite the fact that the work is mainly focused on reactive power compensation, the methodology also deals with power loss issues. In that work, the authors propose three algorithms for EV charging and study the impact of the reactive power injection on the voltage deviation, peak load, and power losses. Similarly, in [109], a $\mathrm{P}-\mathrm{Q}$ control strategy for EV charging stations is proposed, in which EVs are used to compensate the reactive power consumed by wind turbines installed at the distribution system level.

\section{2) Reactive power compensation from EV chargers}

Some works are focused on the EV chargers. For instance, in [98], the authors design a bidirectional EV fast charger that is used to provide reactive power compensation by maintaining voltage regulation and power factor correction. Similarly, in [101] and [110], the authors implement a single-phase on-board bidirectional EV charger, which not only provides energy to the EV batteries, but also provides reactive power support to the grid. In the same way, a control strategy for EV charging and reactive power support using a three-phase off-board EV charger is developed in [104], while in [105], the authors propose a reactive power compensation method for EVs in which the power limit of the system is controlled by establishing an active power priority. An alternative approach is proposed in [102], in which the authors develop an optimal coordinated voltage regulation algorithm including EV, DG, and on-load tap-changer devices. Alternatively, the work in [103] proposes an EV reactive power control, dependent on the voltage. The objective aims to support the loaded phases and mitigate the voltage imbalances of the grid without affecting user comfort.

\section{3) Smart charging/discharging strategies}

Distinct from the aforementioned works, a smart charging/discharging strategy of EV aggregators is developed in [106] to provide services to the grid within an energy management system. A similar approach can be found in [107], in which the authors propose an algorithm for continuous reactive power management by EVs and PV systems, considering technical aspects of the network. Similar results can be found in [99], in which a reactive power management strategy is proposed.

In [108], a grid-supporting EV station is modeled, and the EVs provide active and reactive power support to the grid by controlling the power angle and voltage. The approach in [100] evaluates the capability and cost of providing reactive power support by EVs through a mathematical model that considers the grid's technical constraints and EV user requirements. In [111], voltage control is addressed by managing the reactive power from EVs. A mathematical model is proposed to minimize EV load curtailment, power losses, and load shifting in an implicit way.

Regarding voltage control services, most of the previous studies that address reactive power compensation implicitly address voltage issues, as previously mentioned.

\section{Grid integration of RES}

Along with EVs, RES integration into distribution systems is emerging as a solution to cope with environmental issues. However, it has been demonstrated that a high penetration of RES may cause operational problems to the grid due to its intermittency, bringing additional challenges for DSOs [112].

The joint operation of EVs and RES is a good option for DSOs to deal with these issues. EVs can support the grid operation in different ways, taking into account that some EV-DSS, for instance, are inspired in the services provided by DG units and energy storage devices to TSOs. The services included in this category refer to those in which EVs help to support the integration of RES into the grid by mitigating the intermittent output power from solar energy (PV) or wind energy (WE). EV energy consumption is encouraged during the periods of high RES power production in order to avoid power curtailments. Moreover, when properly managed, EVs can act as storage devices to satisfy the demand when there is a deficit in the power production from RES.

Regarding the services provided by EVs for supporting RES integration into distribution systems, a number of studies examine the large-scale integration of EVs for supporting the integration of WE [113] and PV in power systems [114]-[116]. Despite the fact that WE is usually integrated into the transmission systems, the penetration of wind power at the distribution system level has also been studied in [117], in which demand response strategies are proposed to prevent overvoltage issues. In contrast, solar energy is usually integrated at the distribution system level, at which overvoltage issues are also addressed [118], [119].

\section{1) Joint operation of EVs, WE, and PV systems}

Several approaches have focused on the joint operation of EVs and RES. In [34] and [120], reviews related to the ability of EVs to support RES integration into the distribution system, as well as its economic and environmental impacts, are presented. The work presented in [10] proposes an algorithm for controlling EV charging/discharging to deal with the high penetration of RES. The algorithm considers wind turbines at the medium voltage (MV) level and solar panels at the LV level, and it uses EVs for the grid support, dealing mainly with voltage deviation issues. Similarly, in [121], the authors propose a method for EV charging coordination, in which the EVs are considered responsive loads that can provide reserve services to the grid in order to compensate for renewable power production variability (WE and PV systems). Another approach is proposed in [122], in which a mathematical model is developed to manage the integrated operation of both plug-in EV parking lots and RES. In this work, the technical constraints of the distribution network are partly taken into account.

\section{2) Joint operation of $E V S$ and $P V$ systems}

The authors in [123] develop a control strategy for EV charging/discharging to mitigate PV system impacts: overvoltage during the day, peak load during the evening, and reverse power flows. Similar strategies are proposed in [97], aiming at reducing the PV power curtailment caused by overvoltages under vehicle-to-home scenarios. In the same way, in [96], an algorithm for managing the integrated operation of EVs and PV systems by using EVs as storage devices is proposed. The objective is to maximize the participation of PV systems in ancillary services by using EV storage, considering the EV user requirements and the grid 
constraints. The storage also can help to deal with the peak load at night. In [114]-[116] and [124], storage devices are also included within the operational strategies for EVs and RES.

Even though the approaches in [9], [78], [107], and [109] were described in previous sections, they also address the joint management of EVs and RES in order to improve the operation of the grid.

\section{Limitation/Weakness of the reviewed works and research requirements}

It is clear that most of the previous papers are focused on developing optimal strategies and methods to control the EV charging coordination, aiming to minimize/maximize different objectives named in this manuscript as EV-DSS. However, it could be observed from the literature review that important aspects within the strategies are disregarded or even developed based on unrealistic assumptions, making the implementation of EV-DSS in real-life applications difficult.

\section{1) Price-based strategies in active power control}

Most of the services regarding active power support deal with load congestion and peak demand issues, which may be influenced by price signals and uncontrollable elements, such as socioeconomic and environmental factors. These issues typically appear during the peak hours when not only is the conventional demand consumed, but, at the same time, EVs are being charged. Different strategies can be used for grid congestion management, such as distribution grid capacity market, advance capacity allocation, and dynamic tariffs [112], [125]. Most of the strategies for grid congestion management, for instance, are based on the electricity price behavior, and their main objective is encouraging the energy consumption of EVs during off-peak hours, when the electricity price is lower. In this context, the grid congestion management based on dynamic tariffs provides indirect service to the grid. However, these strategies should be carefully addressed in order to avoid creating new technical problems.

In terms of the peak demand, for example, one must bear in mind that peak hours do not only take place when the electricity price is high. For instance, in distribution systems with a high penetration of RES, the market may face negative prices (low prices) during the daytime. This may result in new peak hours and new technical issues, such as overloading in some feeders or undervoltage problems in some nodes, although the electricity price is still low. For example, Fig. 4 shows a case in Denmark in which the electricity prices reached negative values in the off-peak hours $(12: 00-16: 00)$ during the daytime on May 1, 2017. Lower prices could create new peaks if people are encouraged to charge their EVs during those hours, induced by the RES power production. Therefore, shifting the load to periods with lower electricity prices may be achieved at the expense of creating new peak demands. In the reviewed literature, some of the price-based signal strategies for EV charging/discharging coordination are focused on minimizing operational costs or EV charging costs. Although most of the works consider the technical constraints of the grid, the creation of new peaks is scarcely addressed. Therefore, price-based strategies in active power control should be carefully designed because new and potentially inconvenient peaks may be created.

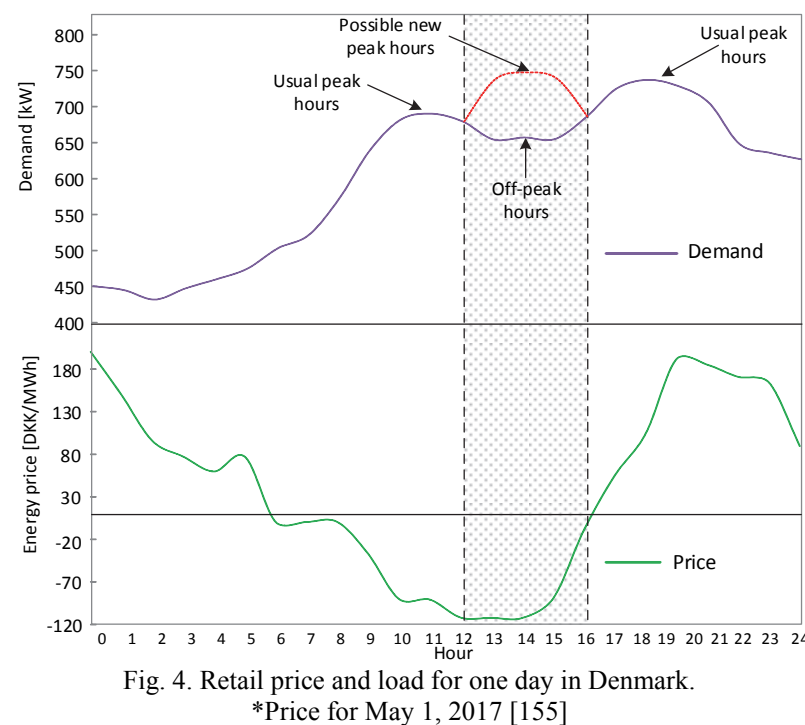

2) Strategies for joint management of EVS and RES.

One way to deal with load congestion and new peak issues is properly managing the joint operation between EVs and RES, which could bring several advantages for DSOs. In the case of PV systems, for instance, it is known that their high penetration in distribution systems may cause new issues for the grid. Congestion, for example, can happen due to a high load or high power production of PVs. Then, DSOs may face overvoltage/undervoltage in some nodes or overloading in the transformers. However, EVs can help to alleviate those problems and to maintain the grid operation within reliable conditions. During the period of high PV production, the EVs can increase charging, even if the price of the electricity is high. In that case, EVs would help with the integration of PV systems into distribution systems by preventing the curtailment or, ultimately, the need of disconnection from the grid. Furthermore, if the EVs do not need to be charged during the periods of high PV production, they can be used as storage devices, as long as their batteries have available capacity, storing the surplus of energy coming from the PVs. This energy can be used during peak hours through V2G technology. There are few studies that consider this as a potential market for EV-DSS, which affords room to propose new strategies for the integrated coordination between EVs and RES in distribution systems.

Although there is room for new investigations on the aforementioned topics, there is still one limitation that strongly affects the implementation of EV-DSS, which is the assumption of market existence for each service, as further discussed below.

3) Assumptions on market existence and economic calculations

Several important aspects, such as the existence of a market; regulations; or economic aspects, e.g., the cost related to these services, are assumed or simply disregarded in most of the literature reviewed. A figurative example is explained as follows: If a strategy is developed to coordinate the charging/discharging of EVs in order to minimize the charging cost from the owner's perspective, a contract between the owner and the other party (probably an aggregator or even the 
DSO) should exist. If the owner is willing to provide services for the DSO, how should the remuneration process be carried out? How should be the contract? How much can the owner earn for providing services for the DSO? How does the market work for each service? Many of these open questions have been addressed at the transmission system level. However, at the distribution system level, all of these topics remain unclear.

In this way, managing the charging/discharging control of EVs as a service for supporting the distribution grid becomes a new paradigm that requires an appropriate regulatory framework that endorses the EV service provision for DSOs [31]. By having a clear concept on it, the current strategies and methods developed to provide EV-DSS can be improved, or new ones can even be proposed to align with the current requirements for realistic implementations. Therefore, further research in market and economic aspects is required in order to ease the implementation of EV-DSS in real applications.

Based on the previous discussion, it can be seen that most of the literature reviewed remains in theoretical implementations. Thus, it would be useful to assess how far the EV-DSS are from being implemented in real applications. Hence, in the following section, an assessment of the maturity of EV-DSS is provided.

\section{IV.ASSESSMENT OF THE MATURITY OF EV-DSS FOR REAL APPLICATIONS}

Based on the background, it is clear that there are many strategies and solutions suggested for EV-DSS. However, since EV-DSS are a new paradigm, DSOs are not yet allowed to acquire services from other stakeholders or to participate in the market. Therefore, it is relevant to assess the implementability of these services in real applications and the possibility of being combined with frequency regulation as a special case. This is done by aiming at encouraging the development of local DSO markets and promoting the active participation of EVs as service providers for DSOs. The base case of frequency controlled normal operation reserve (FNR found in Denmark zone DK2) provided by EVs to the TSOs used for this comparison framework is supported by the real implementation of EV services through the Parker project [24]. The definition of these two comparison parameters is presented as follows:

Implementability: Ease of implementation of EV-DSS in a time horizon when compared with the current implementation of a real-life case of FNR provided by EV to TSOs.

Stackability: Possibility of combining different services. For simplicity, the comparison will be carried out between the categories previously defined in Table II, using a special case of FNR with EV-DSS and the possibility of combining them.

\section{A. Implementability}

The implementability of the EV-DSS is assessed by taking into account three time horizons as shown in Fig. 5: short-term ( 0 -2 years), medium-term (2-5 years), and long-term (over 5 years). The assessment considers aspects such as the information and capabilities that are expected to be available for their implementation, for instance, infrastructure, technology, policies, and market framework, among others.

From Fig. 5, it is possible to observe that FNR is a current service provided by EVs (here and now). However, one has to bear in mind that frequency regulation is a service procured by

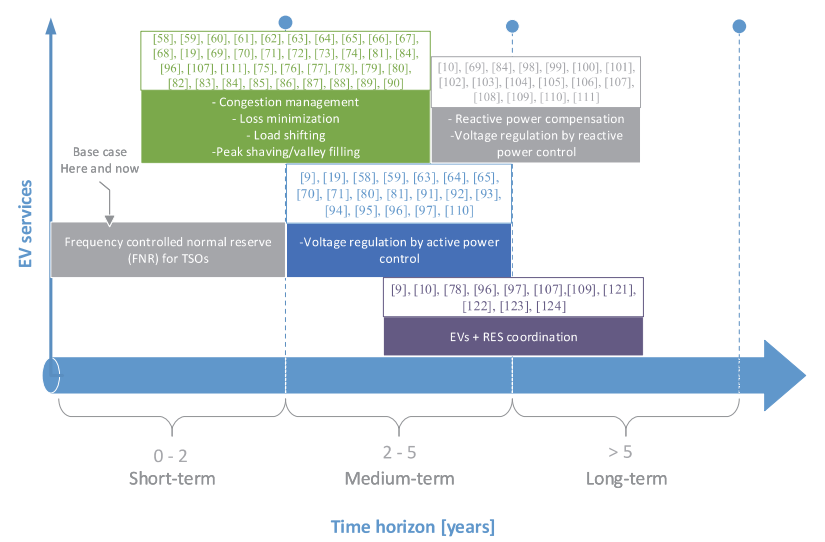

Fig. 5. Assessment of the maturity in the EV-DSS implementation.

TSOs for which there is a complete market framework designed. Comparing this specific case of FNR with EV-DSS, it is possible to observe that, for instance, reactive power compensation is a service that could be implemented in a medium-long-term time horizon. Although new grid codes in Denmark include policies that allow EV chargers to provide reactive power to the grid [126], reactive power support cannot be implemented in the short-term because there is not yet any market for reactive power services. In addition, the majority of $\mathrm{EV}$ chargers are not able to control the reactive power at the connection point.

Congestion management, load shifting, peak shaving, and valley filling could be implemented between a short- and medium-term time horizon, as indicated by some real applications of these services in California, in which the load is shifted, based on EV tariffs [127], [128]. These kinds of services are easier to implement since they are based on controlling active power, for which there are more market framework proposals. However, the application of these services depends on the demand in each market. For this reason, there are still some challenges from the DSO market perspective that should be addressed to definitively establish these services as EV-DSS.

Voltage regulation by active power is in the medium-term time horizon since this is a high-level power quality indicator. Currently, monetary penalties are applied to network operators in case of voltage quality issues. Since there are no markets specifically developed for this service, voltage regulation by active power still needs time to develop an appropriate market structure and become a DSO service. Voltage regulation by reactive power control could be implemented in the medium-long-term time horizon. The grid codes allow EV chargers to provide reactive power, but special equipment capable of providing reactive power (which is not common in current EV chargers) should be specially designed. Some companies are working on implementing this technology on new EV chargers.

EVs + RES integration support for DSOs are services that could be implemented in the medium-long-term time horizon since there are already well-defined market frameworks for service provisions from RES to TSOs. These models can be used as a base to create new markets for the joint operation of EVs and RES as a DSO product. Moreover, there are some real 
TABLE IV

ASSESSMENT OF THE STACKABILITY OF THE EV-DSS PER CATEGORIES BASED ON THE PROPOSED CLASSIFICATION OF TABLE II.

\begin{tabular}{c|c|ccc|c|cc}
\cline { 2 - 6 } Category & $\begin{array}{c}\text { FNR (Special } \\
\text { case) }\end{array}$ & $\begin{array}{c}\text { Active power } \\
\text { support }\end{array}$ & $\begin{array}{c}\text { Reactive } \\
\text { power } \\
\text { support }\end{array}$ & $\begin{array}{c}\text { RES } \\
\text { integration } \\
\text { support }\end{array}$ & $\begin{array}{c}\text { FNR (Special } \\
\text { case) }\end{array}$ & $\begin{array}{c}\text { Active power } \\
\text { support }\end{array}$ & $\begin{array}{c}\text { Reactive } \\
\text { power support }\end{array}$ \\
\hline $\begin{array}{c}\text { integration } \\
\text { support }\end{array}$ \\
\hline Active power support & $\mathrm{H}$ & - & $\mathrm{H}$ & $\mathrm{H}$ & $\mathrm{L}$ & - & $\mathrm{H}$ \\
Reactive power support & $\mathrm{H}$ & - & - & $\mathrm{H}$ & $\mathrm{H}$ & - & $\mathrm{M}$ \\
RES integration support & $\mathrm{H}$ & - & - & - & $\mathrm{M}$ & - & - \\
\hline \hline
\end{tabular}

implementations of services provided by RES. In Germany, for instance, peak reduction and voltage regulation services are being implemented through PV units connected to the distribution network in response to reduced grid tariffs [129]. However, the increasing penetration of PV units causes operational issues to the grid, resulting in grid expansion requirements. EVs can be used to support the integration of RES at the distribution system level under suitable regulations and market frameworks specifically developed or adjusted for such services. A lack of rules and standards for managing this technology and equipment in distribution grids makes it difficult to see the implementation of such services in the near future.

\section{B. Stackability}

The stackability of services is assessed using three levels: high, medium, and low (easy, medium, and difficult), considering two ways of application: scheduled or simultaneous. The possibility of a combination of EV-DSS per category with a special case of FNR and the possibility of combining between them are shown in Table IV. The last column in Table III provides information about the combination of different services in each paper included in the literature review.

The services in Table IV included in the category of active power support, such as congestion management, loss minimization, load shifting, peak shaving, and valley filling, can be easily combined with frequency regulation because these services are mainly constrained by time. This means that they are activated during specific periods of time, e.g., off-peak hours, according to the requirements of the distribution grid. Then, EVs can be used during the remaining time to provide other services, such as frequency regulation, assuming a minimum energy requirement per hour to satisfy the user preferences. However, the combination of these services is limited by the kind of time scheduling, e.g., in the day-ahead (scheduled), intra-day, or real-time (simultaneous). The high-level combination of these services works if they are previously scheduled, because the power modulation is scheduled according to the peak or valley periods. Otherwise, the stackability of services may be reduced because, in the intra-day or real-time market in which simultaneous services may be necessary, continuous power modulation is required. This hinders the combination of services because real-time information is needed, which requires special technology and equipment. Continuous power modulation implies that EVs should be ready at any time to provide specific services, which reduces the possibility of offering other services such as RES integration. However, the combination between FNR and reactive power compensation, and between FNR and voltage regulation by reactive power control qualifies for a high-level since FNR only requires active power modulation and the reactive power could be adjusted correspondingly.

It is possible to see from Table IV that there is a high possibility of combination between FNR and RES integration support since these services also depend on time. EVs can be used during specific periods of time to mitigate the intermittent output power production from RES, and in the remaining time, they can be used to provide other services, such as frequency regulation. However, it could be more difficult to combine these services simultaneously, since frequency regulation requires constant power modulation, and the EV should be ready to provide the service at any time.

Reactive power compensation and voltage regulation by reactive power control could be combined, with most of the services belonging to the category of active power support, regardless of application. It is important to remember that this service is mainly limited by the requirement of special technology and equipment. Thus, it is possible to see that the reactive power provision is far from being implemented in the future, but would actually be mixable with the active power services for DSOs and TSOs.

The stackability of the services belonging to the category of active and reactive power support and to the category of RES integration support is high, as long as the services are prescheduled. This is because RES operation is constrained in time. Then, EVs can be used to support the grid when the RES operation is causing operational challenges, and in other periods of time, they can support the grid problems related to congestion, voltage drops, or overloading, e.g., caused by an increase in conventional demand. Stackability of these services in a simultaneous way is limited to the coincidence in time of operational problems caused by RES operation and those caused by other reasons, e.g., increase in conventional demand. For this reason, plus the demand for special equipment for information exchange in real-time, the stackability of these services qualifies for a medium level.

Despite the fact that some services can be executed simultaneously, prioritization and a sense of compromise should be accounted for between them. For instance, voltage regulation by active and reactive power can be combined with FNR; however, according to the system conditions, it should be defined which of those services has the highest priority.

\section{Challenges and Future Research Suggestions}

In this paper, a classification, summary, and comparison of the works addressing EV services specifically developed to support the distribution grid have been presented. Following the previous analysis, it can be concluded that, although there are certain works addressing EV-DSS, there are still key aspects that need to be tackled. An analysis of these key aspects can identify and highlight open challenges and needs for further 
research in specific areas to move toward realistic implementations of EV-DSS. In this way, challenges and prospects arising from the transition to a new perception of EV-DSS are discussed in this section.

\section{A. Market framework}

There are several market frameworks that are designed according to the requirements and regulations in each country. For instance, the market framework in the US is composed of generation companies, independent system operators (ISOs), and utility companies. In the US market framework, the ISO is the owner of the market. In Europe, the market framework is almost the same; the difference is that the market operator is independent [130]. Differences between market structures in several countries can be found in [26], [27]. According to the European Network of Transmission System Operators for Electricity (ENTSO-E) [131], there are available market frameworks for contemporary ancillary services provided to TSOs, such as frequency containment reserve capacity/energy, frequency restoration reserve capacity/energy (automatic), frequency restoration reserve capacity/energy (manual), and replacement reserves. The types of market frameworks in Europe are based on bilateral markets, free offers, hybrid markets, mandatory offers, mandatory provision, mandatory provision without reservation, and organized markets. In a bilateral market, for instance, a grid user and TSO negotiate a contract for the offered service and price/price system [131].

Regarding the market framework for EV-DSS, it could be mentioned that clearly, there is not much consensus regarding EV-DSS in the contemporary literature. At the moment, there is a market for frequency regulation services, but these services are usually required for TSOs. In contrast, at the distribution system level, there are market framework proposals for services such as congestion management and voltage regulation by using active power control [132], while for load shifting, peak shaving, and valley filling, they have not been proposed yet. Because there is no commercial market defined for reactive power support, the economic incentive is focused on active power support. Several works address loading and voltage regulation services by modulating the EV active power. Nonetheless, some EV users may not enable this service due to the delay in the charging process, which affects their comfort. Furthermore, the communication infrastructure and users' privacy become additional limitations in the service provision [31].

Regarding reactive power support, there is a high potential for using EVs to provide voltage regulation by injecting reactive power into the grid. One of the reasons for using EVs on that purpose is that reactive power does not affect the charging process of the EVs, which maintains user comfort. Few works have addressed reactive power control strategies [98], [100], [101], [103]-[105]. This is a new area that could be explored, despite some limitations, especially those related to the requirements of special chargers with $\mathrm{V} 2 \mathrm{G}$ technology, along with a market framework.

In this way, from the market framework point of view, market design for EV-DSS is a potential topic for further research since most of the DSOs are not allowed yet to acquire services from other parties or to participate in the market [31]. New market models that enable active and reactive EV-DSS, such as load shifting, peak shaving, valley filling, voltage regulation, and reactive power control at the distribution system level, can be investigated and proposed to enforce the participation of DSOs in the market as an active player.

\section{B. Economic aspects}

According to the literature review, it could be mentioned that there is a lack of information in terms of the economic aspects of EV-DSS. Most of the studies focus on EV coordination in order to address loading and voltage issues, disregarding the details of the economic aspects, such as the revenues for providing services to the DSOs. One possible reason could be that these calculations depend highly on the regulation policies and socioeconomic and environmental conditions of each country. It is worth mentioning that the main incentive for the $\mathrm{EV}$ owners to allow active power control is the minimization of the EV charging cost. Usually, these strategies are based on electricity prices and involve the EVs being charged during periods of low electricity prices. However, most of the studies focus on developing strategies, methodologies, and algorithms for EV charging coordination while disregarding the economic aspects of EV-DSS.

One important issue regarding the economic aspects of EV-DSS is the definition of the price signals, so that the results benefit all stakeholders. The work presented in [133] proposes a market framework, introducing a design for an EV charging billing process based on a two-price model in order to incorporate EV services into distribution systems. According to the simulations carried out in [133] using a real distribution system in Denmark, one EV user does not receive much money per year for providing services to the DSOs, considering one single charging event per EV per day. It is important to mention that the estimation of the revenues for the DSOs is not easy to assess because there are several parties involved in the retail electricity market with a corresponding revenue distribution.

On the other hand, although there is not a market framework for service provision at the distribution system level, and even though there is no information regarding the economic aspects associated with them, a good starting point could be the inclusion of these services as part of the grid codes in the future. In Denmark, for instance, EV chargers should be able to provide reactive power to the grid [126].

For these reasons, more research on this topic is encouraged in an attempt to answer many questions that remain unanswered. For instance, who will be able to provide flexibility to the DSOs? How can consumers be encouraged to provide services while being profitable? How much may consumers earn by providing services for DSOs? How should the grid operating rules be modified to include EV-DSS? What should the regulation framework be? It is worth mentioning that the answers to these questions depend on each country and the special needs of each DSO. These matters have been poorly addressed in contemporary literature. Therefore, economic evaluations, including aspects such as benefit analysis for all stakeholders and possible remuneration strategies for service providers, are still open for research.

\section{EV battery degradation caused by EV services}

Because EVs can offer grid support by controlling the charging/discharging of their batteries, the operational 
strategies developed for this control may affect the battery life. Factors mainly related to the operation of the EV, such as charge transfer, charging rate, and average SOC, affect the battery life span. When providing services, there is a higher probability of incurring on more EV charge and discharge cycles, and the charging rate should be modulated according to the control signal, e.g., the frequency signal, which may reduce the life span of the battery. Hence, it is expected that service provision may have a detrimental impact on battery degradation.

Some studies have focused on this matter. In [5], the authors analyze the effect on the EV battery life caused by the grid support and propose measures to reduce these negative impacts. In [134], a battery degradation cost model is developed based on the cost incurred by EV users as a result of the charging/discharging activities associated with participation in V2G programs. A similar approach can be found in [135], in which only the battery discharging degradation cost is taken into account to calculate the effect of providing V2G services. The works presented in [136] and [137] also address the battery degradation effects caused by EV service provision. Therefore, the impact on battery degradation is an important issue that should be always considered and assessed within the strategies developed to provide EV-DSS, because the integration of battery degradation costs may change the charging/discharging strategies. Moreover, it is necessary to answer questions in regard to economic calculations: How much damage is caused by providing services? How is this damage compensated? Who is responsible for the payment? Hence, this topic becomes an interesting potential field for future research.

A comparison of the hourly energy exchanged (charged and discharged) of one EV with and without providing services can be seen in Fig. 6. Fig. 6a shows the possible conventional daily energy exchanged for one EV Nissan LEAF with a battery capacity of $24 \mathrm{kWh}$. Considering the average travel distance per day in Denmark, which is approximately $40 \mathrm{~km} \mathrm{[138],} \mathrm{it}$ results in a daily energy consumption for driving of approximately $8 \mathrm{kWh}$. Furthermore, it is assumed that the EV is used for traveling to work and that it is connected in a residential area for charging, using a slow charger with power rated at $3.6 \mathrm{~kW}$. Assuming an unknown initial SOC of the battery and also assuming that the charging process will only finish when the car is disconnected or fully charged (according to the user preferences) it can be seen from Fig. 6a that the energy discharged only depends on the energy required for driving and that the charging rate is stable. Fig. $6 \mathrm{~b}$ shows the total energy exchanged per hour of the same EV Nissan Leaf providing FNR to Energinet, the TSO in Denmark, through an ENEL V2G charger with $10 \mathrm{~kW}$ of power rated capacity during one complete day (data from Parker [24], a project in which a real demonstration of FNR provided by an EV fleet in Denmark is being carried out [139]). It can be seen from Fig. 6b that during some hours (e.g., hour 1 and 4 ) there are energy charged and discharged at the same time. This is because the frequency signal is measured with one-second resolution, and due to its high fluctuation and uncertain behavior, the system may face over and under frequencies from one second to another. This may result in charging and discharging actions during the same hour, which means that the EV may consume or inject power from/to the grid during one hour.
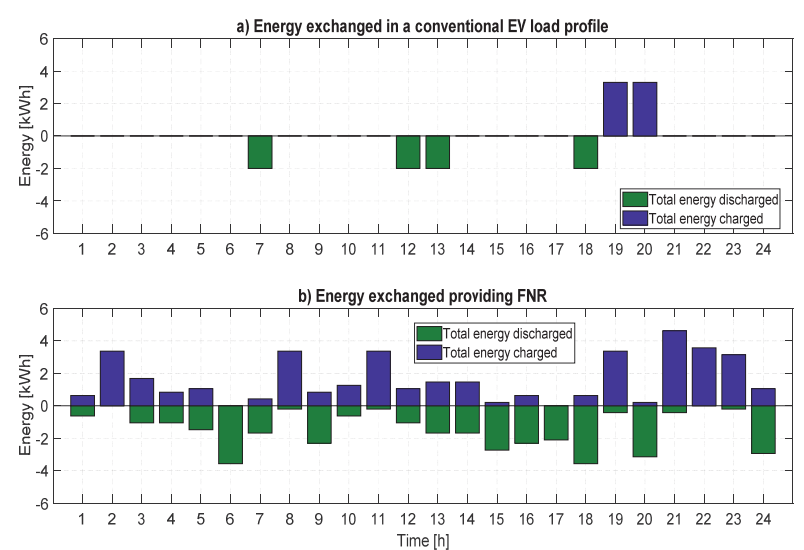

Fig. 6. Daily energy exchanged for one car.

Regulation period during one weekend day (Sunday, September 10, 2017).

The differences between the charging rates for both cases are depicted in Fig. 6. As can be seen, the energy exchanged in the EV transportation load profile is less than when the car is providing FNR services to the grid. It is worth mentioning that the amount of energy charged or discharged in the case of FNR fluctuates hour by hour. Since the energy exchanged is directly related to the depth of discharge of the battery, the service provision may result in more battery degradation.

\section{Impacts of TSO service provision by EVS on distribution grids}

Since TSOs are responsible for grid stability and security, TSOs are responsible for maintaining the balance between electricity consumption and production by procuring ancillary services, such as frequency and voltage regulation. Frequency regulation is one of the most common services procured by TSOs for grid balancing, and EVs are pointed out as a prominent option to provide this service due to the manageable nature of their loads, availability, bidirectional power capacity, and quick response time [140]. However, considering that the EVs are mainly connected to the distribution network, ancillary services provision for TSOs may affect the distribution grid to which EVs are connected; that automatically results in a conflict of interest between DSOs and TSOs [141], [142]. This topic has been scarcely studied in the literature; therefore, it deserves more attention, since EVs have been becoming more popular as new service providers in recent years.

The preliminary results from Parker [24] allow us to further discuss the possible conflicts between TSOs and DSOs. Fig. 7 shows the real operation of one EV (the same described in Fig. 6b) providing FNR to Energinet during one complete day. Fig.7 depicts the power provided by the EV with one-second resolution in the case of over frequencies, where down-regulation is required and the charging mode is activated, and in the case of under frequencies, where up-regulation is required and the discharging mode is activated. As can be seen, high power fluctuations occur during the day, caused by the charging and discharging actions resulting from the frequency signals. On one hand, it has been already demonstrated that a high penetration of EVs in distribution systems causes detrimental impacts on the network. On the other hand, if this power fluctuation is extrapolated to a huge number of EVs 


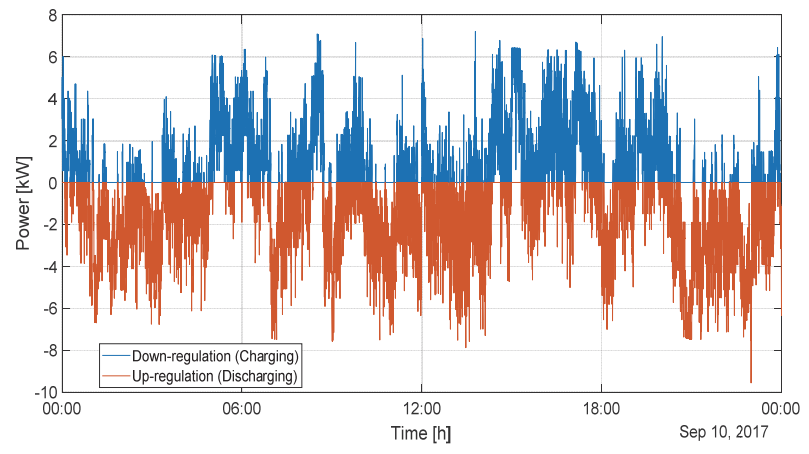

Fig. 7. Power fluctuation of one car during one complete day. Regulation period during one weekend day (Sunday, September 10, 2017).

connected at the distribution level, technical issues (e.g., overloading in the transformers, line congestion, voltage drops, or phase unbalances) are anticipated. Hence, the effect of this power fluctuation on the distribution grid should be taken into account when developing strategies for service provision by EVs to TSOs. In the same way, the fact that service provision from EVs to TSOs may cause conflicts between DSOs and TSOs leads us to anticipate that the service provision from EVs to DSOs will cause conflicts between DSOs and other stakeholders, such as aggregators, balance responsible parties, or even the end users (according to the market structure).

Considering the lack of strong research addressing this topic, and based on the preliminary results of the Parker project, it is possible to claim that further investigations on the conflicts that may arise from the EV service provision are required in an attempt to answer several open questions: How would DSOs be compensated for the operational problems caused by the TSO service provision? Who will pay for the damages caused in the distribution grid? Are there stacked services that can solve problems for different parties at the same time (for instance, DSOs and TSOs)? The answers to these questions can ease the implementation of DSO services in real applications.

\section{E. Emerging EV technologies and their impact on distribution grids}

EVs are part of different technologies involved in smart cities, especially those used to reduce green-house emissions and deal with environmental issues. Other emerging technologies, such as connected and autonomous vehicles, are being incorporated in smart cities as a way to deal with traffic and transportation issues. In a future transportation electrification scenario, the combination of these two technologies may bring potential benefits, but also challenges, in several aspects [143], [144]. Autonomous vehicles and EVs are complementary from a technology point of view [145][147]. For instance, EVs could be easier for auto driving due to the electric components [148].

Autonomous and connected EVs (ACEVs) may have the capability to manage many functionalities, such as SOC, the charging power, and the charging/discharging time, without deteriorating user comfort [144]. To this aim, ACEVs need to make use of communication tools, that allow them to find charging facilities. For instance, in [149], the authors develop a communication tool for vehicles to localize access points online with improved connectivity signals. Similarly, a methodology for the optimal allocation of wireless power transfer systems for autonomous EVs is presented in [150]. In [151], the authors develop a software that allows for communication between the database and a group of drones, which could be adapted for the operation of ACEV fleets. ACEVs may use similar technologies to manage energy functions in an automatized and optimized way and to enable communication between involved parties (e.g., ACEVs, users, operating system, transportation utilities, electricity companies, aggregators, etc.).

As a result, it is expected that ACEVs will be integrated in the power system due to their energy requirements, which may bring additional challenges to distribution systems. ACEVs' load will represent an increase in the energy demand, which may provoke operational problems with the grid, similar to those provoked by current EVs. However, the ACEVs' capabilities may be advantageous for DSOs or aggregators in terms of flexibility, since the charging/discharging of ACEVs could be more easily programmed using communication technologies to support the system operation through different DSO services. In this way, DSOs may cope with challenges arising from uncertain variables related to EV user behavior, since it is difficult to predict when and where a private EV owner will connect the car to charge its battery.

Few works have analyzed the impacts of the ACEVs' integration in power systems [144], [152], [153]. Moreover, similar to the current EVs, a large-scale implementation of ACEVs and the service provision to DSOs must still overcome many barriers related to the policies, agreements, infrastructure, and technology [146], [154]. Considering the growing penetration of emerging EV technologies and the lack of research in the context of DSO services, further investigations are required in this regard to pave the way toward an optimized integration of emerging $\mathrm{EV}$ technologies in distribution systems. Additionally, it is worth exploring the possibility of adapting the methods already proposed in the current literature (those developed to optimize the integration of EVs in the power system) to cope with the integration of ACEVs in distribution grids.

\section{CONCLUSIONS}

This paper presented a review of the recent literature focusing on distribution system services provided by EVs (EV-DSS). It was found that there is not an agreement regarding the classification of the services provided by EVs for DSOs, which could be because of the novelty of the concept at the distribution level; therefore, a new classification of EV-DSS was proposed, including three main categories: active power support, reactive power support, and renewable energy source (RES) integration support. A description of the services, basic formulations, and the main contributions of the reviewed papers were presented. This information provided an overview of the traditional methods and allowed us to identify weaknesses in the control strategies as a means to encourage exploring new ones, aligned with the current requirements for the realistic implementation of services from EVs for DSOs.

Additionally, a comparison framework of the work developed in the academic research with a real-life application of EV services using a specific case of frequency regulation service was proposed. Using the proposed framework, it is 
possible to assess the implementability of EV-DSS, i.e., how far the EV-DSS are from being implemented, and the stackability of the EV-DSS, i.e., how easily EV-DSS services can be combined with frequency regulation and other potential services.

This information can help researchers to identify new potential areas of research that need to be addressed to move toward the realistic implementation of EV-DSS. It was found that active power services have a high chance of being implemented in the short-term, while reactive power compensation is a service that could be implemented in a medium-long-term time horizon because of the technical and market limitations. It was also found that most of the services belonging to the category of active power support can be highly combined with frequency regulation and with reactive power services under specific conditions. As a last step, a comprehensive discussion regarding the challenges and prospects of the EV-DSS was presented. It included key topics such as market framework design, economic assessment, battery degradation, and impacts of TSO service provision by EVs on distribution grids. It is concluded that, although there were certain strategies and solutions proposed to address EV-DSS, most of these strategies were far from being implemented in real applications because they fail on assuming possible roles for DSOs as an active market player or even assuming the existence of a market for each service. Considering these aspects within the EV-DSS strategies may significantly increase their implementation in real-life cases. Moreover, it may help to promote the active participation of EVs as a services provider for DSOs and to stimulate the creation of regulations, standards, and market framework for DSO services.

In addition, although most of the aspects that remain unclear for moving toward realistic implementations of EV-DSS are related to market and regulatory barriers, other important barriers may also exist, e.g., the impact on the distribution grid and battery degradation caused by the EV service provision to any party of the whole power system. Thus, these kinds of topics are suggested as future research directions associated with EV-DSS.

\section{REFERENCES}

[1] M. S. Kumar and S. T. Revankar, "Development scheme and key technology of an electric vehicle: An overview," Renew. Sustain. Energy Rev., vol. 70, no. July 2016, pp. 1266-1285, 2017.

[2] A. Dubey and S. Santoso, "Electric vehicle charging on residential distribution systems: Impacts and mitigations," IEEE Access, vol. 3. pp. 1871-1893, 2015.

[3] J. Xiong, K. Zhang, Y. Guo, and W. Su, "Investigate the impacts of PEV charging facilities on integrated electric distribution system and electrified transportation system," IEEE Trans. Transp. Electrif., vol. 1, no. 2, pp. 178-187, Aug. 2015.

[4] K. Zafred, J. Nieto-Martin, and E. Butans, "Electric vehicles - effects on domestic low voltage networks," in 2016 IEEE International Energy Conference (ENERGYCON), 2016, pp. 1-6.

[5] G. Lacey, G. Putrus, and E. Bentley, "Smart EV charging schedules: supporting the grid and protecting battery life," IET Electr. Syst. Transp., vol. 7, no. 1, pp. 84-91, 2017.

[6] E. Veldman and R. A. Verzijlbergh, "Distribution grid impacts of smart electric vehicle charging from different perspectives," IEEE Trans. Smart Grid, vol. 6, no. 1, pp. 333-342, 2015.

[7] J. Quirós-Tortós, L. F. Ochoa, S. W. Alnaser, and T. Butler, "Control of EV charging points for thermal and voltage management of LV networks," IEEE Trans. Power Syst., vol. 31, no. 4, pp. 3028-3039,
2016.

[8] H. N. T. Nguyen, C. Zhang, and A. Mahmud, "Optimal coordination of $\mathrm{G} 2 \mathrm{~V}$ and $\mathrm{V} 2 \mathrm{G}$ to support power grids with high penetration of renewable energy," IEEE Trans. Transp. Electrif., vol. 1, no. 2, pp. 188195, 2015.

[9] E. Akhavan-Rezai, M. F. Shaaban, E. F. El-Saadany, and F. Karray, "Managing demand for plug-in electric vehicles in unbalanced LV systems with photovoltaics," IEEE Trans. Ind. Informatics, vol. 13, no. 3, pp. 1057-1067, Jun. 2017.

[10] R. A. Kordkheili, B. Bak-Jensen, J. R. Pillai, M. Savaghebi, and J. M. Guerrero, "Managing high penetration of renewable energy in MV grid by electric vehicle storage," in 2015 International Symposium on Smart Electric Distribution Systems and Technologies (EDST), 2015, pp. 127132.

[11] J. Lin, J. J. Q. Yu, K.-C. Leung, and V. O. K. Li, "Optimal scheduling with vehicle-to-grid ancillary services," Energy Syst. Electr. Hybrid Veh., vol. 2, p. 395, 2016.

[12] C. Le Floch, E. Kara, and S. Moura, "PDE Modeling and control of electric vehicle fleets for ancillary services: A discrete charging case," IEEE Trans. Smart Grid, 2016.

[13] M. Hou, Y. Zhao, and X. Ge, "Optimal scheduling of the plug-in electric vehicles aggregator energy and regulation services based on grid to vehicle," Int. Trans. Electr. Energy Syst., vol. 27, no. 6, pp. 1-12, 2017.

[14] S. I. Vagropoulos, D. K. Kyriazidis, and A. G. Bakirtzis, "Real-time charging management framework for electric vehicle aggregators in a market environment," IEEE Trans. Smart Grid, vol. 7, no. 2, pp. 948957, 2016.

[15] J. Tomić and W. Kempton, "Using fleets of electric-drive vehicles for grid support," J. Power Sources, vol. 168, no. 2, pp. 459-468, 2007.

[16] G. Wenzel, M. Negrete-Pincetic, D. E. Olivares, J. MacDonald, and D. S. Callaway, "Real-time charging strategies for an electric vehicle aggregator to provide ancillary services," IEEE Trans. Smart Grid, vol. In press, pp. 1-1, 2017.

[17] K. Knezović, M. Marinelli, P. Codani, and Y. Perez, "Distribution grid services and flexibility provision by electric vehicles: A review of options," in Proc. Universities Power Eng. Conf., 2015.

[18] J. Hu, H. Morais, T. Sousa, and M. Lind, "Electric vehicle fleet management in smart grids: A review of services, optimization and control aspects," Renew. Sustain. Energy Rev., vol. 56, pp. 1207-1226, 2016.

[19] S. Sarabi, A. Davigny, V. Courtecuisse, Y. Riffonneau, and B. Robyns, "Potential of vehicle-to-grid ancillary services considering the uncertainties in plug-in electric vehicle availability and service/localization limitations in distribution grids," Appl. Energy, vol. 171, pp. 523-540, 2016.

[20] K. M. Tan, V. K. Ramachandaramurthy, and J. Y. Yong, "Integration of electric vehicles in smart grid: A review on vehicle to grid technologies and optimization techniques," Renew. Sustain. Energy Rev., vol. 53, pp. 720-732, 2016.

[21] N. Shaukat, B. Khan, S. M. Ali, C. A. Mehmood, J. Khan, U. Farid, M. Majid, S. M. Anwar, M. Jawad, and Z. Ullah, "A survey on electric vehicle transportation within smart grid system," Renew. Sustain. Energy Rev., vol. 81, pp. 1329-1349, 2018.

[22] L. Rubino, C. Capasso, and O. Veneri, "Review on plug-in electric vehicle charging architectures integrated with distributed energy sources for sustainable mobility," Appl. Energy, vol. 207, pp. 438-464, 2017.

[23] K. Mahmud, G. E. Town, S. Morsalin, and M. J. Hossain, "Integration of electric vehicles and management in the internet of energy," Renew. Sustain. Energy Rev., vol. 82, pp. 4179-4203, 2018.

[24] "DANMARK, NISSAN NEWSROOM. Nissan, Enel and Nuvve operate world's first fully commercial vehicle-to-grid hub in Denmark," $2016 . \quad$ [Online]. Available: https://newsroom.nissan-europe.com/dk/da-dk/media/pressreleases/149 191. [Accessed: 19-Nov-2017].

[25] E. Hirst and B. Kirby, Ancillary services. Oak Ridge, Tennessee: Oak Ridge National Laboratory, 1996.

[26] Y. G. Rebours, D. S. Kirschen, M. Trotignon, and S. Rossignol, "A survey of frequency and voltage control ancillary services - Part I: Technical features," IEEE Trans. Power Syst., vol. 22, no. 1, pp. 350357, 2007.

[27] Y. G. Rebours, D. S. Kirschen, M. Trotignon, and S. Rossignol, “A survey of frequency and voltage control ancillary services - Part II: Economic features," IEEE Trans. Power Syst., vol. 22, no. 1, pp. 358366, 2007.

[28] R. A. Verzijlbergh, M. O. W. Grond, Z. Lukszo, J. G. Slootweg, and M. 
D. Ilic, "Network impacts and cost savings of controlled EV charging," IEEE Trans. Smart Grid, vol. 3, no. 3, pp. 1203-1212, 2012.

[29] J. García-Villalobos, I. Zamora, J. I. San Martín, F. J. Asensio, and V. Aperribay, "Plug-in electric vehicles in electric distribution networks: A review of smart charging approaches," Renew. Sustain. Energy Rev., vol. 38 , pp. 717-731, 2014

[30] T. Sousa, T. Soares, H. Morais, R. Castro, and Z. Vale, "Simulated annealing to handle energy and ancillary services joint management considering electric vehicles," Electr. Power Syst. Res., vol. 136, pp. 383-397, 2016.

[31] K. Knezović, M. Marinelli, A. Zecchino, P. B. Andersen, and C. Traeholt, "Supporting involvement of electric vehicles in distribution grids: Lowering the barriers for a proactive integration," Energy, vol. 134, pp. 458-468, 2017.

[32] RTE, "Report on the balancing mechanism: August 2015." Réseau de transport d'électricité, 01-Aug-2015.

[33] J. Jefferson, A. Saldanha, J. Jefferson, A. Saldanha, E. Machado, A. Paula, C. De Mello, A. Paula, and C. De Mello, "Control strategies for smart smart charging discharging of plug-in electric vehicles," in World's largest Science, Technology \& Medicine Open Access book publisher, InTech., I. N. Da Silva and R. Andrade Flauzino, Eds. InTech, 2016.

[34] M. Ghofrani, E. Detert, N. N. Hosseini, A. Arabali, N. Myers, and N. Phasith, "V2G Services for renewable integration," in Modeling and Simulation for Electric Vehicle Applications, M. A. Fakhfakh, Ed. InTech, 2016.

[35] Z. Li, M. Chowdhury, P. Bhavsar, and Y. He, "Optimizing the performance of vehicle-to-grid (V2G) enabled battery electric vehicles through a smart charge scheduling model," Int. J. Automot. Technol., vol. 16 , no. 5, pp. 827-837, 2015 .

[36] M. Leo, K. Kavi, H. Anders, and B. Moss, "Ancillary service revenue opportunities from electric vehicles via demand response," 2011.

[37] E. Sortomme and M. A. El-Sharkawi, "Optimal scheduling of vehicle-to-grid energy and ancillary services," IEEE Trans. Smart Grid, vol. 3, no. 1, pp. 351-359, 2012.

[38] A. Janjic and L. Z. Velimirovic, "Optimal scheduling of utility electric vehicle fleet offering ancillary services," ETRI J., vol. 37, no. 2, pp 273-282, 2015.

[39] M. Ansari, A. T. Al-Awami, E. Sortomme, and M. A. Abido, "Coordinated bidding of ancillary services for vehicle-to-grid using fuzzy optimization," IEEE Trans. Smart Grid, vol. 6, no. 1, pp. 261270, Jan. 2015.

[40] Z. Zhou, C. Sun, R. Shi, Z. Chang, S. Zhou, and Y. Li, "Robust energy scheduling in vehicle-to-grid networks," IEEE Netw., vol. 31, no. 2, pp 30-37, 2017.

[41] D. R. Melo, A. Trippe, H. B. Gooi, and T. Massier, "Robust electric vehicle aggregation for ancillary service provision considering battery aging," IEEE Trans. Smart Grid, vol. 9, no. 3, pp. 1728-1738, 2018.

[42] M. Vahedipour-Dahraie, H. Rashidizaheh-Kermani, H. Najafi, A. Anvari-Moghaddam, and J. Guerrero, "Coordination of EVs participation for load frequency control in isolated microgrids," Appl. Sci., vol. 7, no. 6, pp. 1454-5101, 2017.

[43] M. F. M. Arani and Y. Mohamed, "Cooperative control of wind power generator and electric vehicles for microgrid primary frequency regulation," IEEE Trans. Smart Grid, p. (To be published), 2017.

[44] M. H. Khooban, "Secondary load frequency control of time-delay stand-alone microgrids with electric vehicles," IEEE Trans. Ind. Electron., vol. 65, no. 9, pp. 7416-7422, 2018.

[45] M. H. Khooban, T. Niknam, M. Shasadeghi, T. Dragicevic, and F. Blaabjerg, "Load frequency control in microgrids based on a stochastic noninteger controller," IEEE Trans. Sustain. Energy, vol. 9, no. 2, pp 853-861, 2018.

[46] P. Palensky and D. Dietrich, "Demand side management: Demand response, intelligent energy systems, and smart loads," IEEE Trans. Ind Informatics, vol. 7, no. 3, pp. 381-388, 2011.

[47] M. Zhou, Y. Gao, and G. Li, "Study on improvement of available transfer capability by demand side management," in 2008 Third International Conference on Electric Utility Deregulation and Restructuring and Power Technologies, 2008.

[48] T. Logenthiran, D. Srinivasan, and T. Z. Shun, "Demand side management in smart grid using heuristic optimization," IEEE Trans. Smart Grid, vol. 3, no. 3, pp. 1244-1252, 2012.

[49] Federal energy regulatory commision, "Assessment of demand response and advanced mitering," 2010.

[50] C. Vivekananthan, Y. Mishra, G. Ledwich, and F. Li, "Demand response for residential appliances via customer reward scheme," IEEE Trans. Smart Grid, vol. 5, no. 2, pp. 809-820, 2014.

[51] C. W. Gellings, "The concept of demand-side management for electric utilities," Proc. IEEE, vol. 73, no. 10, pp. 1468-1470, 1985.

[52] S. Saini, "Demand-side management module," Sustain. Energy Regul. Policymaking Africa, pp. 1-100, 2007.

[53] "ABB." [Online] http://new.abb.com/substations/energy-storage-applications/load-leveli ng. [Accessed: 18-Jul-2017].

[54] D. S. Carr, "Lead/acid batteries in U.S.A. load-levelling applications," J. Power Sources, vol. 31, no. 1, pp. 297-310, 1990.

[55] J. Quirós-Tortós, L. F. Ochoa, and B. Lees, "A statistical analysis of EV charging behavior in the UK," in 2015 IEEE PES Innovative Smart Grid Technologies Latin America, ISGT LATAM 2015, 2016, pp. 445-449.

[56] A. N. M. M. Haque, P. H. Nguyen, W. L. Kling, and F. W. Bliek, "Congestion management in smart distribution network," in 2014 49th International Universities Power Engineering Conference (UPEC), 2014, pp. 1-6.

[57] Dansk Energi; Universidad Carlos III de Madrid; Tampere University of Technology, "IDE4L: Deliverable 5.2/3: Congestion Management in Distribution Networks," 2015.

[58] K. Knezović, S. Martinenas, P. B. Andersen, A. Zecchino, and M. Marinelli, "Enhancing the role of electric vehicles in the power grid: Field validation of multiple ancillary services," IEEE Trans. Transp. Electrif., vol. 3, no. 1, pp. 201-209, 2017.

[59] J. Hu, H. Morais, T. Sousa, S. You, and R. D'Hulst, "Integration of electric vehicles into the power distribution network with a modified capacity allocation mechanism," Energies, vol. 10, no. 2, 2017.

[60] J. Hu, A. Saleem, S. You, L. Nordström, M. Lind, and J. Østergaard, “A multi-agent system for distribution grid congestion management with electric vehicles," Eng. Appl. Artif. Intell., vol. 38, pp. 45-58, 2015.

[61] F. Wu and R. Sioshansi, "A two-stage stochastic optimization model for scheduling electric vehicle charging loads to relieve distribution-system constraints," Transp. Res. Part B Methodol., vol. 102, pp. 55-82, 2017.

[62] J. Hu, S. You, M. Lind, and J. Østergaard, "Coordinated charging of electric vehicles for congestion prevention in the distribution grid," IEEE Trans. Smart Grid, vol. 5, no. 2, pp. 703-711, 2014

[63] J. Romero-Ruiz, J. Pérez-Ruiz, S. Martin, J. A. Aguado, and S. De La Torre, "Probabilistic congestion management using EVs in a smart grid with intermittent renewable generation," Electr. Power Syst. Res., vol. 137, pp. 155-162, 2016.

[64] S. Hajforoosh, M. A. S. Masoum, and S. M. Islam, "Online optimal variable charge-rate coordination of plug-in electric vehicles to maximize customer satisfaction and improve grid performance," Electr. Power Syst. Res., vol. 141, pp. 407-420, 2016.

[65] S. Mocci, N. Natale, F. Pilo, and S. Ruggeri, "Multi-agent control system for the exploitation of vehicle to grid in active LV networks," in CIRED Workshop 2016, 2016, pp. 1-4.

[66] Z. Liu, Q. Wu, S. S. Oren, S. Huang, R. Li, and L. Cheng, "Distribution locational marginal pricing for optimal electric vehicle charging through chance constrained mixed-integer programming," IEEE Trans. Smart Grid, vol. 9, no. 2, pp. 644-654, 2018.

[67] Z. Ma, N. Yang, S. Zou, and Y. Shao, "Charging coordination of Plug-In electric vehicles in distribution networks with capacity constrained feeder lines," IEEE Trans. Control Syst. Technol., vol. 26, no. 5, pp. 1917-1924, 2018

[68] S. Huang and Q. Wu, "Dynamic subsidy method for congestion management in distribution networks," IEEE Trans. Smart Grid, vol. 9, no. 3 , pp. $2140-2151,2018$

[69] S. Weckx and J. Driesen, "Load balancing with EV chargers and PV inverters in unbalanced distribution grids," IEEE Trans. Sustain. Energy, vol. 6, no. 2, pp. 635-643, 2015.

[70] A. Kavousi-Fard, M. A. Rostami, and T. Niknam, "Reliability-oriented reconfiguration of vehicle-to-grid networks," IEEE Trans. Ind. Informatics, vol. 11, no. 3, pp. 682-691, 2015.

[71] T. Sousa, H. Morais, Z. Vale, and R. Castro, "A multi-objective optimization of the active and reactive resource scheduling at a distribution level in a smart grid context," Energy, vol. 85, pp. 236-250, Jun. 2015.

[72] M. Esmaili and A. Goldoust, "Multi-objective optimal charging of plug-in electric vehicles in unbalanced distribution networks," Int. J. Electr. Power Energy Syst., vol. 73, pp. 644-652, 2015.

[73] N. Banol Arias, J. F. Franco, M. Lavorato, and R. Romero, "Metaheuristic optimization algorithms for the optimal coordination of plug-in electric vehicle charging in distribution systems with distributed 
generation,” Electr. Power Syst. Res., vol. 142, pp. 351-361, 2017.

[74] A. Hajizadeh and M. R. Kikhavani, "Coordination of bidirectional charging for plug-in electric vehicles in smart distribution systems," Electr. Eng., vol. 100, no. 2, pp. 1085-1096, 2018.

[75] F. Rassaei, W. S. Soh, and K. C. Chua, "Demand response for residential electric vehicles with random usage patterns in smart grids," IEEE Trans. Sustain. Energy, vol. 6, no. 4, pp. 1367-1376, 2015.

[76] M. A. Fotouhi Ghazvini, J. Soares, O. Abrishambaf, R. Castro, and Z. Vale, "Demand response implementation in smart households," Energy Build., vol. 143, pp. 129-148, 2017.

[77] M. A. López, S. De La Torre, S. Martín, and J. A. Aguado, "Demand-side management in smart grid operation considering electric vehicles load shifting and vehicle-to-grid support," Int. J. Electr. Power Energy Syst., vol. 64, pp. 689-698, 2015.

[78] M. Aziz, T. Oda, T. Mitani, Y. Watanabe, and T. Kashiwagi, "Utilization of electric vehicles and their used batteries for peak-load shifting," Energies, vol. 8, no. 5, pp. 3720-3738, 2015.

[79] R. Mehta, D. Srinivasan, A. M. Khambadkone, J. Yang, and A. Trivedi, "Smart charging strategies for optimal integration of plug-in electric vehicles within existing distribution system infrastructure," IEEE Trans. Smart Grid, vol. 9, no. 1, pp. 299-312, 2018.

[80] Y. Nie, X. Wang, and K. W. E. Cheng, "Multi-area self-adaptive pricing control in smart city with EV user participation," IEEE Trans. Intell. Transp. Syst., vol. 19, no. 7, pp. 2156-2164, 2018.

[81] E. L. Karfopoulos, K. A. Panourgias, and N. D. Hatziargyriou, "Distributed coordination of electric vehicles providing V2G regulation services," IEEE Trans. Power Syst., vol. 31, no. 4, pp. 2834-2846, 2016.

[82] M. Singh, K. Thirugnanam, P. Kumar, and I. Kar, "Real-time coordination of electric vehicles to support the grid at the distribution substation level," IEEE Syst. J., vol. 9, no. 3, pp. 1000-1010, 2015.

[83] M. A. Hussain and M. J. Lee, "Mobility incorporated vehicle-to-grid (V2G) optimization for uniform utilization in smart grid based power distribution network," in Smart Grid Inspired Future Technologies: First International Conference, SmartGIFT, 2017, pp. 3-15.

[84] N. Leemput, F. Geth, J. Van Roy, J. Büscher, and J. Driesen, "Reactive power support in residential LV distribution grids through electric vehicle charging," Sustain. Energy, Grids Networks, vol. 3, pp. 24-35, 2015.

[85] M. J. E. Alam, K. M. Muttaqi, and D. Sutanto, "A controllable local peak-shaving strategy for effective utilization of PEV battery capacity for distribution network support," IEEE Trans. Ind. Appl., vol. 51, no. 3, pp. 2030-2037, 2015.

[86] E. Ramos Muñoz, G. Razeghi, L. Zhang, and F. Jabbari, "Electric vehicle charging algorithms for coordination of the grid and distribution transformer levels," Energy, vol. 113, pp. 930-942, Oct. 2016.

[87] G. Binetti, A. Davoudi, D. Naso, B. Turchiano, and F. L. Lewis, "Scalable real-time electric vehicles charging with discrete charging rates," IEEE Trans. Smart Grid, vol. 6, no. 5, pp. 2211-2220, 2015.

[88] L. Jian, Y. Zheng, and Z. Shao, "High efficient valley-filling strategy for centralized coordinated charging of large-scale electric vehicles," Appl. Energy, vol. 186, pp. 46-55, 2017.

[89] W. Zhang, D. Zhang, B. Mu, L. Y. Wang, Y. Bao, J. Jiang, and H. Morais, "Decentralized electric vehicle charging strategies for reduced load variation and guaranteed charge completion in regional distribution grids," Energies, vol. 10, no. 2, 2017.

[90] M. C. Kisacikoglu, F. Erden, and N. Erdogan, "Distributed control of PEV charging based on energy demand forecast," IEEE Trans. Ind. Informatics, vol. 14, no. 1, pp. 332-341, 2018

[91] O. Sundström and C. Binding, "Flexible charging optimization for electric vehicles considering distribution grid constraints," IEEE Trans. Smart Grid, vol. 3, no. 1, pp. 26-37, 2012.

[92] P. Richardson, D. Flynn, and A. Keane, "Optimal charging of electric vehicles in low-voltage distribution systems," IEEE Trans. Power Syst., vol. 27 , no. 1 , pp. $268-279,2012$

[93] H. F. Farahani, "Improving voltage unbalance of low-voltage distribution networks using plug-in electric vehicles," J. Clean. Prod., vol. 148, pp. 336-346, 2017.

[94] S. Martinenas, K. Knezović, and M. Marinelli, "Management of power quality issues in low voltage networks using electric vehicles: Experimental validation," IEEE Trans. Power Deliv., vol. 32, no. 2, pp. 971-979, 2017.

[95] J. E. Cardona, J. C. López, and M. J. Rider, "Decentralized electric vehicles charging coordination using only local voltage magnitude measurements," Electr. Power Syst. Res., vol. 161, pp. 139-151, 2018.

[96] R. Ahmadi Kordkheili, S. A. Pourmousavi, M. Savaghebi, J. M.
Guerrero, and M. H. Nehrir, "Assessing the potential of plug-in electric vehicles in active distribution networks," Energies, vol. 9, no. 1, 2016.

[97] H. Kikusato, K. Mori, S. Yoshizawa, Y. Fujimoto, H. Asano, Y. Hayashi, A. Kawashima, S. Inagaki, and T. Suzuki, "Electric vehicle charge-discharge management for utilization of photovoltaic by coordination between home and grid energy management systems," IEEE Trans. Smart Grid, p. (To be published).

[98] J. Y. Yong, V. K. Ramachandaramurthy, K. M. Tan, and N. Mithulananthan, "Bi-directional electric vehicle fast charging station with novel reactive power compensation for voltage regulation," Int. J. Electr. Power Energy Syst., vol. 64, pp. 300-310, 2015.

[99] S. Pirouzi, M. A. Latify, and G. R. Yousefi, "Investigation on reactive power support capability of PEVS in distribution network operation," in ICEE 2015 - Proceedings of the 23rd Iranian Conference on Electrical Engineering, 2015, vol. 10, pp. 1591-1596.

[100] M. N. Mojdehi, M. Fardad, and P. Ghosh, "Technical and economical evaluation of reactive power service from aggregated EVs," Electr. Power Syst. Res., vol. 133, pp. 132-141, 2016.

[101] M. C. Kisacikoglu, M. Kesler, and L. M. Tolbert, "Single-phase on-board bidirectional PEV charger for V2G reactive power operation," IEEE Trans. Smart Grid, vol. 6, no. 2, pp. 767-775, 2015.

[102] M. A. Azzouz, M. F. Shaaban, and E. F. El-Saadany, "Real-time optimal voltage regulation for distribution networks incorporating high penetration of PEVs," IEEE Trans. Power Syst., vol. 30, no. 6, pp. 3234-3245, Nov. 2015.

[103] K. Knezović and M. Marinelli, "Phase-wise enhanced voltage support from electric vehicles in a Danish low-voltage distribution grid," Electr. Power Syst. Res., vol. 140, pp. 274-283, 2016.

[104] M. Kesler, M. C. Kisacikoglu, and L. M. Tolbert, "Vehicle-to-grid reactive power operation using plug-in electric vehicle bidirectional offboard charger," IEEE Trans. Ind. Electron., vol. 61, no. 12, pp. 67786784,2014

[105] J. Gallardo-lozano, E. Romero-cadaval, S. Member, V. Miñambres-marcos, D. Vinnikov, T. Jalakas, and H. Hõimoja, "Grid reactive power compensation by using electric Vehicles," in 2014 Electric Power Quality and Supply Reliability Conference (PQ), 2014, pp. 19-24.

[106] M. P. Anand, S. Golshannavaz, W. Ongsakul, and A. Rajapakse, "Incorporating short-term topological variations in optimal energy management of MGs considering ancillary services by electric vehicles," Energy, vol. 112, pp. 241-253, 2016.

[107] O. Gandhi, W. Zhang, C. D. Rodríguez-Gallegos, D. Srinivasan, and T. Reindl, "Continuous optimization of reactive power from PV and EV in distribution system," in 2016 IEEE Innovative Smart Grid Technologies - Asia (ISGT-Asia), 2016, pp. 281-287.

[108] K. Thirugnanam and P. Kumar, "Fuzzy based active and reactive power support to the distribution network using electric vehicles," in 2015 IEEE International Transportation Electrification Conference (ITEC), 2015, pp. 1-6.

[109] C. Wu, H. Akhavan-Hejazi, H. Mohsenian-Rad, and J. Huang, "PEV-based P-Q control in line distribution networks with high requirement for reactive power compensation," in 2014 IEEE PES Innovative Smart Grid Technologies Conference, ISGT 2014, 2014.

[110] M. Restrepo, J. Morris, M. Kazerani, and C. A. Cañizares, "Modeling and testing of a bidirectional smart charger for distribution system EV integration," IEEE Trans. Smart Grid, vol. 9, no. 1, pp. 152-162, 2018.

[111] J. Wang, G. R. Bharati, S. Paudyal, O. Ceylan, B. P. Bhattarai, and K. S. Myers, "Coordinated electric vehicle charging with reactive power support to distribution grids," IEEE Trans. Ind. Informatics, p. (To be published).

[112] B. Muruganantham, R. Gnanadass, and N. P. Padhy, "Challenges with renewable energy sources and storage in practical distribution systems," Renew. Sustain. Energy Rev., vol. 73, pp. 125-134, 2017.

[113] H. N. T. Nguyen, C. Zhang, and J. Zhang, "Dynamic demand control of electric vehicles to support power grid with high penetration level of renewable energy," IEEE Trans. Transp. Electrif., vol. 2, no. 1, pp. 66$75,2016$.

[114] J. Ugirumurera and Z. Haas, "Optimal sizing of a completely green charging system for electric vehicles," IEEE Trans. Transp. Electrif.

[115] Y. T. Liao and C. N. Lu, "Dispatch of EV charging station energy resources for sustainable mobility," IEEE Trans. Transp. Electrif., vol. 1, no. 1, pp. 86-93, 2015.

[116] D. Wu, H. Zeng, C. Lu, and B. Boulet, "Two-stage energy management for office buildings with workplace EV charging and renewable energy," IEEE Trans. Transp. Electrif., vol. 3, no. 1, pp. 225-237, 2017. 
[117] S. Chalise, H. R. Atia, B. Poudel, and R. Tonkoski, "Impact of active power curtailment of wind turbines connected to residential feeders for overvoltage prevention," IEEE Trans. Sustain. Energy, vol. 7, no. 2, pp. 471-479, 2016.

[118] M. Karimi, H. Mokhlis, K. Naidu, S. Uddin, and A. H. A. Bakar, "Photovoltaic penetration issues and impacts in distribution network - A review," Renew. Sustain. Energy Rev., vol. 53, pp. 594-605, 2016.

[119] S. Hashemi and J. Østergaard, "Methods and strategies for overvoltage prevention in low voltage distribution systems with PV," IET Renew. Power Gener., vol. 11, no. 2, pp. 205-214, 2017.

[120] D. B. Richardson, "Electric vehicles and the electric grid: A review of modeling approaches, Impacts, and renewable energy integration,' Renew. Sustain. Energy Rev., vol. 19, pp. 247-254, Mar. 2013.

[121] A. Zakariazadeh, S. Jadid, and P. Siano, "Integrated operation of electric vehicles and renewable generation in a smart distribution system," Energy Convers. Manag., vol. 89, pp. 99-110, 2015.

[122] M. Shafie-khah, P. Siano, D. Z. Fitiwi, N. Mahmoudi, and J. P. S. Catalão, "An innovative two-level model for electric vehicle parking lots in distribution systems with renewable energy," IEEE Trans. Smart Grid, vol. 9, no. 2, pp. 1506-1520, 2018.

[123] M. J. E. Alam, K. M. Muttaqi, and D. Sutanto, "Effective utilization of available PEV battery capacity for mitigation of solar PV impact and grid support with integrated V2G functionality," IEEE Trans. Smart Grid, vol. 7, no. 3, pp. 1562-1571, 2016.

[124] C. Sabillon, J. F. Franco, M. J. Rider, and R. Romero, "Joint optimal operation of photovoltaic units and electric vehicles in residential networks with storage systems: A dynamic scheduling method," Int. J. Electr. Power Energy Syst., vol. 103, pp. 136-145, 2018.

[125] P. Bach Andersen, J. Hu, and K. Heussen, "Coordination strategies for distribution grid congestion management in a multi-actor, multi-objective setting," in IEEE PES Innovative Smart Grid Technologies Conference Europe, 2012.

[126] ENERGINET, "Regulations for grid connection," Technical regulation 3.3.1 for battery plants, 2017.

[127] Edison International Company, "Electric Vehicle Rates," Southern California, 2018. [Online]. Available: https://www.sce.com/wps/portal/home/residential/electric-cars/resident ial-rates/. [Accessed: 15-Jan-2018].

[128] San Diego Gas \& Electric Company, "EV Rates," SDGE, 2016. [Online]. Available: https://www.sdge.com/clean-energy/ev-rates. [Accessed: 15-Jan-2018].

[129] E. Bayer, "Report on the German power system," Brussels, Belgium, 2015.

[130] T. V. Jensen, "Exploring market models for a european electricity grid with a high penetration of renewable energy sources," Technical University of Denmark, 2017.

[131] ENTSO-E, "Survey on ancillary services procurement and electricity balancing market design," 2017.

[132] C. Zhang, Y. Ding, N. C. Nordentoft, P. Pinson, and J. Østergaard, "FLECH: A Danish market solution for DSO congestion management through DER flexibility services," J. Mod. Power Syst. Clean Energy, vol. 2, no. 2, pp. 126-133, Jun. 2014.

[133] A. O. Gadea, "Technical investigation and economic assessment of DSO based services from electric vehicles," Technical University of Denmark, Risø, Roskilde, Denmark, 2017.

[134] H. Farzin, M. Fotuhi-Firuzabad, and M. Moeini-Aghtaie, "A practical scheme to involve degradation cost of lithium-ion batteries in vehicle-to-grid applications," IEEE Trans. Sustain. Energy, vol. 7, no. 4, pp. 1730-1738, 2016.

[135] R. C. Leou, "Optimal charging/discharging control for electric vehicles considering power system constraints and operation costs," IEEE Trans Power Syst., vol. 31, no. 3, pp. 1854-1860, 2016.

[136] D. Wang, J. Coignard, T. Zeng, C. Zhang, and S. Saxena, "Quantifying electric vehicle battery degradation from driving vs. vehicle-to-grid services," J. Power Sources, vol. 332, pp. 193-203, 2016.

[137] K. Darcovich, S. Recoskie, H. Ribberink, F. Pincet, and A. Foissac, "Effect on battery life of vehicle-to-home electric power provision under Canadian residential electrical demand," Appl. Therm. Eng., vol. 114, pp. 1515-1522, 2017.

[138] H. Christiansen and B. Z. Skougaard, "Documentation of the Danish National Travel Survey: DTU Transport,” 2015.

[139] N. Banol Arias, S. Hashemi, P. Bach Andersen, C. Træholt, and R. Romero, "V2G Enabled EVs Providing Frequency Containment Reserves: Field Results," in 2017 IEEE Int. Conf. Ind. Technol (ICIT), 2018 .
[140] E. Romero-Cadaval, F. Barrero-González, E. González-Romera, and M.-I. Milanés-Montero, "Using plug-in electric vehicles to implement ancillary services in smart distribution grids," in Plug In Electric Vehicles in Smart Grids: Integration Techniques, S. Rajakaruna, F. Shahnia, and A. Ghosh, Eds. Singapore: Springer Singapore, 2015, pp. 309-349.

[141] A. Zecchino, K. Knezović, and M. Marinelli, "Identification of conflicts between transmission and distribution system operators when acquiring ancillary services from electric vehicles," in 2017 IEEE PES Innovative Smart Grid Technologies Conference Europe, 2017.

[142] H. Gerard, E. I. R. Puente, and D. Six, "Coordination between transmission and distribution system operators in the electricity sector: A conceptual framework," Util. Policy, vol. 50, pp. 40-48, 2018.

[143] D. Milakis, B. Van Arem, and B. Van Wee, "Policy and society related implications of automated driving: A review of literature and directions for future research," J. Intell. Transp. Syst. Technol. Planning, Oper., vol. 21, no. 4, pp. 324-348, 2017.

[144] R. Iacobucci, B. McLellan, and T. Tezuka, "Modeling shared autonomous electric vehicles: Potential for transport and power grid integration," Energy, vol. 158, pp. 148-163, 2018.

[145] D. Strugar, R. Hussain, M. Mazzara, V. Rivera, J. Lee, and R. Mustafin, "On M2M Micropayments: A case study of electric autonomous vehicles," Comput. Res. Repos., 2018.

[146] A. Nikitas, I. Kougias, E. Alyavina, and E. Njoya Tchouamou, "How can autonomous and connected vehicles, electromobility, BRT, hyperloop, shared use mobility and mobility-as-a-service shape transport futures for the context of smart cities?," Urban Sci., vol. 1, no. 36, pp. 1-21, 2017.

[147] T. D. Chen, K. M. Kockelman, and J. P. Hanna, "Operations of a shared, autonomous, electric vehicle fleet: Implications of vehicle \& charging infrastructure decisions," Transp. Res. Part A Policy Pract., vol. 94, pp. 243-254, 2016

[148] R. MCCAULEY, "Why autonomous and electric vehicles are inextricably linked," Goverment Tecnhology, 2017. [Online]. Available: http://www.govtech.com/fs/why-autonomous-and-electric-vehicles-are -inextricably-linked.html. [Accessed: 04-Sep-2018].

[149] D. Wu, Q. Liu, Y. Li, J. A. McCann, A. C. Regan, and N. Venkatasubramanian, "Adaptive lookup of open wifi using crowdsensing," IEEE/ACM Trans. Netw., vol. 24, no. 6, pp. 3634-3647, 2016

[150] V.-D. Doan, H. Fujimoto, T. Koseki, T. Yasuda, H. Kishi, and T. Fujita, "Simultaneous optimization of speed profile and allocation of wireless power transfer system for autonomous driving electric vehicles," IEEJ J. Ind. Appl., vol. 7, no. 2, pp. 189-201, 2018.

[151] D. Wu, D. I. Arkhipov, M. Kim, C. L. Talcott, A. C. Regan, J. A. McCann, and N. Venkatasubramanian, "ADDSEN: Adaptive data processing and dissemination for drone swarms in urban sensing," IEEE Trans. Comput., vol. 66, no. 2, pp. 183-198, 2017.

[152] Z. Yi, J. Smart, and M. Shirk, "Energy impact evaluation for eco-routing and charging of autonomous electric vehicle fleet: Ambient temperature consideration," Transp. Res. Part C Emerg. Technol., vol. 89, pp. 344363, 2018.

[153] A. Y. S. Lam, J. J. Q. Yu, Y. Hou, and V. O. K. Li, "Coordinated autonomous vehicle parking for vehicle-to-grid services: Formulation and distributed algorithm," IEEE Trans. Smart Grid, vol. 9, no. 5, pp. 4356-4366, 2018.

[154] D. J. Fagnant and K. Kockelman, "Preparing a nation for autonomous vehicles: Opportunities, barriers and policy recommendations," Transp. Res. Part A Policy Pract., vol. 77, pp. 167-181, 2015.

[155] "Nord Pool," Spot day-ahead electricity market. [Online]. Available: http://www.nordpoolspot.com/Market-data1/Elspot/Area-Prices/DK/H ourly/?view=table. [Accessed: 28-Jul-2017]. 


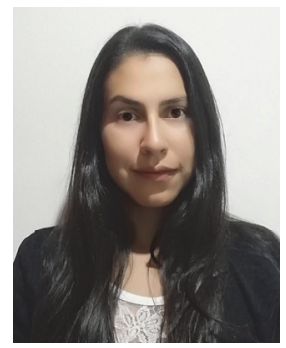

Nataly Bañol Arias received the B.Sc. degree in Production Engineering from the Universidad Tecnológica de Pereira, Colombia in 2012 and the M.Sc. degree in Electrical Engineering from the São Paulo State University (UNESP), Ilha Solteira, Brazil, in 2015. She is currently pursuing the Ph.D. degree in Electrical Engineering at UNESP, Ilha Solteira, Brazil. Her areas of research are the development of methodologies for the optimization, planning, and control of electrical power systems.

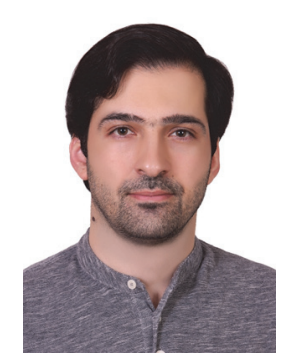

Seyedmostafa Hashemi (S'09, M'16) received the B.Sc. and M.Sc. degree (first class hons.) from Isfahan University of Technology, Isfahan, Iran, in 2006 and 2009, respectively, and the $\mathrm{PhD}$ degree from the Technical University of Denmark in 2016, all in electric power engineering. Since 2016, he has been a Postdoc at the Center for Electric Power and Energy, Technical University of Denmark. His research interests include battery and EV services in distribution systems, and grid integration of renewable energy sources.

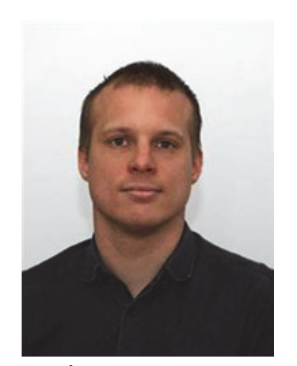

Peter Bach Andersen, $\mathrm{PhD}$, works as senior scientific researcher at the Centre for Electric Power and Energy, part of the Technical University of Denmark (DTU). His main area of research is on how grid integrated electric vehicles may support a stable, economic power system based on renewable energy. Peter has acted as principle investigator in two national projects - currently the Parker project (www.parker-project.com) in collaboration with vehicle manufacturers. He is also a board member of the Danish EV Alliance (Trade organization) and the advisory board of Copenhagen electric (The Capital Region of Denmark) as to support national R\&D efforts in this area.

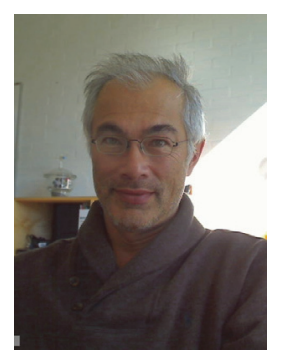

Chresten Traholt received the M.Sc. degree in electrical engineering in 1987 and the Ph.D. degree in materials science in 1994, both from the Technical University of Denmark, Lyngby, Denmark. He worked as research scientist at the Technical University Delft, the Netherlands as well as several years of experience with the superconductor cable industry. He is currently associated professor at the Center for Electric Power and Energy, Department of Electrical Engineering, Technical University of Denmark. His current fields of interest include smart grids, renewable energy, electric vehicles and superconductivity.

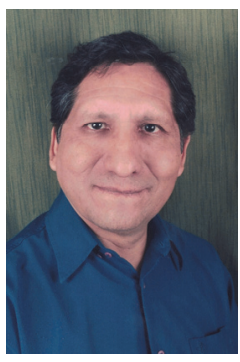

Rubén Romero (M'93-SM'08) received the B.Sc. and P.E. degrees from the National University of Engineering, Lima, Perú, in 1978 and 1984, respectively, and the M.Sc. and $\mathrm{Ph} . \mathrm{D}$. degrees from the Universidade Estadual de Campinas, Campinas, Brazil, in 1990 and 1993, respectively. He is currently a Professor of electrical engineering with the Universidade Estadual Paulista "Julio de Mesquita Filho," Ilha Solteira, Brazil. His research interests include methodologies for the optimization, planning, and control of electrical power systems; applications of artificial intelligence in power system; and operations research. 